\title{
ON THE EULER CHARACTERISTICS OF CERTAIN MODULI SPACES OF 1-DIMENSIONAL SUBSCHEMES
}

\section{A Dissertation}

presented to

the Faculty of the Graduate School

University of Missouri

In Partial Fulfillment

of the Requirements for the Degree

Doctor of Philosophy

by

MAZEN M. ALHWAIMEL

Dr. Zhenbo Qin, Dissertation Supervisor 
The undersigned, appointed by the Dean of the Graduate School, have examined the dissertation entitled

\section{ON THE EULER CHARACTERISTICS OF CERTAIN MODULI SPACES OF 1-DIMENSIONAL SUBSCHEMES}

presented by Mazen M. Alhwaimel, a candidate for the degree of Doctor of Philosophy of Mathematics, and hereby certify that in their opinion it is worthy of acceptance.

Professor Zhenbo Qin

Professor Xinghe Wang

Professor Dana Weston

Professor Qi Zhang 


\section{ACKNOWLEDGEMENTS}

I would like to thank my thesis advisor professor Zhenbo Qin, for his guidance and patience throughout the years I worked as a graduate student in mathematics department at the University of Missouri. His wisdom, motivation, knowledge, and support have been all my strength over the past years. I would also like to thank my committee members professors Xinghe Wang, Dana Weston, Qi Zhang whose helps were very valuable as I was preparing for my comprehensive exams and for my thesis. My gratitude also extends to professors Dan Edidin, Adam Helfer with whom I learned a lot of geometry and algebraic geometry. I would like also to express my sincere gratitude to professor Carlo Morpurgo from whom I learned Analysis when I first studied the subject, and while I was preparing for my qualifying exam. I would like to thank my family, my brothers Saud, and Saad. I also thank my friends Abdulaziz, Abdullah, Faisal,Haithem, Omar, Ibrahim, and Muhammad who have always supported me. I would like to thank the Math Department at Qassim University for their endless help while I was a student. Finally, I would like to thank

professor AbdulRahman Al-Hussein from Qassim University who has always been encouraging me. 


\section{Contents}

$\begin{array}{ll}\text { Acknowledgements } & \text { ii }\end{array}$

Abstract $\quad$ V

1 Introduction 1

1.1 The goal of this thesis . . . . . . . . . . . . . . 1

1.2 Organization of the thesis . . . . . . . . . . . . 3

2 Moduli spaces $\quad 5$

2.1 Basic definitions and constructions . . . . . . . . . . . . 5

2.2 Hilbert scheme and Grothendieck Quot scheme . . . . . . . . . . 9

2.2.1 Hilbert scheme .................. 9

2.2.2 Quot scheme ........................ 11

$3 \quad$ Virtual Hodge polynomials and Euler characteristics $\quad 14$

4 Torus actions on $\operatorname{Hilb}^{n}\left(\mathbb{C}^{r}, O\right) \quad 16$

4.1 -dimensional partitions . . . . . . . . . . . . . 16

4.2 Generating series of plane partitions and torus action . . . . . . . 17

4.3 Important relation . . . . . . . . . . . . . . . . . . . . . . 19

$5 \quad$ The moduli spaces $\mathfrak{I}_{n}(X, \beta)$ and $\mathfrak{M}_{d, n}$ 
5.1 Description of $\mathfrak{I}_{n}(X, \beta)$ and $\mathfrak{M}_{d, n} \ldots \ldots \ldots \ldots$

5.2 Various bijective morphisms . . . . . . . . . . . . . . . 32

6 Reducing the problem and the computation of $\chi\left(\mathfrak{M}_{(2), n}\right) \quad 37$

6.1 Reduction to the local model $\mathbb{C}^{r-1} \times C \ldots \ldots$. . . . . . . 37

6.2 Reduction to the punctual cases . . . . . . . . . . . . . . . . 42

6.3 Torus actions on $\mathfrak{M}_{\xi, n, L, O}^{\mathbb{C}^{r}} \ldots \ldots \ldots \ldots \ldots \ldots$

7 The computation of $\chi\left(\mathfrak{M}_{\left(1^{2}\right), n}\right)$

8 Euler characteristics of $\mathfrak{M}_{2, n} \quad 57$

$\begin{array}{ll}\text { Bibliography } & 60\end{array}$

$\begin{array}{ll}\text { Vita } & 62\end{array}$ 
On the Euler characteristics of certain moduli spaces of 1-dimensional closed subschemes

Mazen M Alhwaimel

Dr. Zhenbo Qin, Dissertation Supervisor

\begin{abstract}
Generalizing the ideas in [LQ] and using virtual Hodge polynomials as well as torus actions, we compute the Euler characteristics of some moduli spaces of 1-dimensional closed subschemes when the ambient smooth projective variety admits a Zariskilocally trivial fibration to a codimension- 1 base. As a consequence, we partially verify a conjecture of W.-P. Li and Qin [LQ]. We also calculate the generating function for the number of certain punctual 3-dimensional partitions, which is used to compute the above Euler characteristics.
\end{abstract}




\section{Chapter 1}

\section{Introduction}

\subsection{The goal of this thesis}

The study of the Euler characteristics of the moduli spaces of 1-dimensional closed subschemes in a smooth projective variety $X$ has been of great interests in recent years. The motivation comes from its relation with the study of Donaldson-Thomas invariants and its interplay with Gromov-Wittin invariants [DT, MNOP1, MNOP2, KLQ, LQ, Tho]. In [LQ], W.-P. Li and Qin proposed the following conjecture which is the analogue to those in [MNOP1, MNOP2] regarding Donaldson-Thomas invariants in dimension 3.

Conjecture 1.1. Let $X$ be a smooth projective variety, and let $\mathfrak{I}_{n}(X, \beta)$ be the moduli space of 1-dimensional closed subschemes $Z$ of $X$ satisfying (5.1.1), and let $X^{[n]}$ be the Hilbert scheme of length- $n$ 0-dimensional closed subschemes of $X$. Then the reduced partition function of the Euler characteristics

$$
\frac{\sum_{n} \chi\left(\Im_{n}(X, \beta)\right) q^{n}}{\sum_{n=0}^{+\infty} \chi\left(X^{[n]}\right) q^{n}}
$$

is a rational function of $q$, and is invariant under $q \rightarrow 1 / q$ when $K_{X}=0$.

Conjecture 1.1 was studied in [LQ] when $X$ admits a Zariski-locally trivial fibration $\mu: X \rightarrow S$ where $S$ is a smooth projective variety, and the fibers are smooth 
irreducible curves of genus $g$. Letting $\beta \in H_{2}(X ; \mathbb{Z})$ be the class of a fiber of $\mu$, it was proved in [LQ] that Conjecture 1.1 holds if $2 \leq \operatorname{dim} X \leq 3$ or $K_{X}=0$.

In this paper, we will continue the investigation of Conjecture 1.1 by generalizing the ideas in [LQ]. Using the same notations from the previous paragraph, put

$$
\mathfrak{M}_{d, n}=\mathfrak{I}_{d(1-g)+n}(X, d \beta)
$$

for $d, n \geq 0$. An element of the moduli space $\mathfrak{M}_{d, n}$ consists of $d$ fibers (possibly nonreduced counting with multiplicities) of $\mu$ together with $n$ points (possibly embedded in the fibers) of $X$. Our main result is the following.

Theorem 1.2. Assume that $X$ admits a Zariski-locally trivial fibration $\mu: X \rightarrow S$ such that the fibers are smooth irreducible curves of genus $g$. Let $r=\operatorname{dim}(X) \geqslant 2$. Then, the partition function $\sum_{n=0}^{+\infty} \chi\left(\mathfrak{M}_{2, n}\right) q^{n}$ is equal to

$$
\begin{gathered}
\frac{\chi(S)^{2}-\chi(S)}{2} \cdot \sum_{n=0}^{+\infty} \chi\left(X^{[n]}\right) q^{n} \cdot\left(\frac{\sum_{n=0}^{+\infty} \widetilde{P}_{r}(n) q^{n}}{\sum_{n=0}^{+\infty} P_{r}(n) q^{n}}\right)^{4-4 g} \\
+(r-1) \cdot \chi(S) \cdot \sum_{n=0}^{+\infty} \chi\left(X^{[n]}\right) q^{n} \cdot\left(\frac{\sum_{n=0}^{+\infty} A_{r}(n) q^{n}}{\sum_{n=0}^{+\infty} P_{r}(n) q^{n}}\right)^{2-2 g} .
\end{gathered}
$$

In the above, $P_{r}(n)$ denotes the number of $r$-dimensional partitions of $n$, and $\widetilde{P}_{r}(n), A_{r}(n)$ denote the number of $r$-dimensional partitions of $n$ punctured at the subsets $\mathcal{I}=\{(0, \ldots, 0)\},\{(0, \ldots, 0),(1,0, \ldots, 0)\} \subset\left(\mathbb{Z}_{\geq 0}\right)^{r-1}$ respectively (see Definition 4.1 for details). The generating function of the Euler characteristics of the Hilbert scheme $X^{[n]}$ has been calculated in [Che, ES, Go1] (see also [Go2, Qin]):

$$
\sum_{n=0}^{+\infty} \chi\left(X^{[n]}\right) q^{n}=\left(\sum_{n=0}^{+\infty} P_{r}(n) q^{n}\right)^{\chi(X)}
$$

Under our assumptions about $X$ and $\mu, K_{X}=0$ forces $g=1$. Also,

$$
\sum_{n} \chi\left(\mathfrak{I}_{n}(X, 2 \beta)\right) q^{n}=\sum_{n=0}^{+\infty} \chi\left(\mathfrak{I}_{2(1-g)+n}(X, 2 \beta)\right) q^{2(1-g)+n}=q^{2(1-g)} \cdot \sum_{n=0}^{+\infty} \chi\left(\mathfrak{M}_{2, n}\right) q^{n} .
$$


Corollary 1.3. Assume that $X$ admits a Zariski-locally trivial fibration $\mu: X \rightarrow S$ such that the fibers are smooth irreducible curves. Let $\operatorname{dim}(X) \geqslant 2$ and $K_{X}=0$. Then, Conjecture 1.1 holds for the reduced partition function

$$
\frac{\sum_{n} \chi\left(\mathfrak{I}_{n}(X, 2 \beta)\right) q^{n}}{\sum_{n=0}^{+\infty} \chi\left(X^{[n]}\right) q^{n}} .
$$

By definition, $P_{2}(n)=\widetilde{P}_{2}(n)=A_{2}(n)$. By Lemma 7.5 in [LQ] (see (4.2.7)),

$$
\frac{\sum_{n=0}^{+\infty} \widetilde{P}_{3}(n) q^{n}}{\sum_{n=0}^{+\infty} P_{3}(n) q^{n}}=\frac{1}{1-q} .
$$

Moreover, by Proposition 4.4 below, when $|q|<1 / 3$, we have

$$
\frac{\sum_{n=0}^{+\infty} A_{3}(n) q^{n}}{\sum_{n=0}^{+\infty} P_{3}(n) q^{n}}=\frac{1}{1-q} \cdot \frac{1}{1-q^{2}} .
$$

Corollary 1.4. Assume that $X$ admits a Zariski-locally trivial fibration $\mu: X \rightarrow S$ such that the fibers are smooth irreducible curves. Let $2 \leq \operatorname{dim}(X) \leq 3$ and $|q|<1 / 3$. Then, Conjecture 1.1 holds for the reduced partition function (1.1.5).

\subsection{Organization of the thesis}

Our main goal as stated in the Section 1.1 is to prove Theorem 1.2. In order to do so, and to make the thesis self-contained as much as possible, I will do the following. In Chapter 2, I will closely follow the book $[\mathrm{HM}]$ for the introduction of moduli problems and how to construct moduli spaces. Next, I will reproduce the construction of Hilbert scheme and Grothendieck Quot scheme analogue to the one found in [Qin, Nit]. To this end, we will be equipped with the necessary concepts and definitions to proceed to prove Theorem 1.2 as follows. First of all, we decompose the moduli space $\mathfrak{M}_{2, n}$ into the disjoint union of two locally closed subsets $\mathfrak{M}_{(2), n}$ and $\mathfrak{M}_{\left(1^{2}\right), n}$. The subset $\mathfrak{M}_{(2), n}$ (respectively, $\mathfrak{M}_{\left(1^{2}\right), n}$ ) consists of the elements in $\mathfrak{M}_{2, n}$ corresponding to those 
1-dimensional closed subschemes $Z$ of $X$ such that the support of the 1-dimensional component $\Theta_{Z}$ of $Z$ is a single fiber of $\mu$ (respectively, is the union of two distinct fibers of $\mu$ ). On one hand, since the support of $\Theta_{Z}$ with $[Z] \in \mathfrak{M}_{\left(1^{2}\right), n}$ is the union of two distinct fibers of $\mu$, the computation of $\chi\left(\mathfrak{M}_{\left(1^{2}\right), n}\right)$ can be easily reduced to $\chi\left(\mathfrak{M}_{1, n}\right)$ which has already been computed in [LQ]. The contribution of $\chi\left(\mathfrak{M}_{\left(1^{2}\right), n}\right)$ gives rise to the term (1.1.2). On the other hand, since the support of $\Theta_{Z}$ with $[Z] \in \mathfrak{M}_{(2), n}$ is a single fiber of $\mu$, the ideas in [LQ] can be generalized to compute the Euler characteristic of $\mathfrak{M}_{(2), n}$. Roughly speaking, we will use the properties of virtual Hodge polynomials to reduce the calculation of $\chi\left(\mathfrak{M}_{(2), n}\right)$ to the relevant moduli space over the local model $\mathbb{C}^{r-1} \times C$ where $C$ denotes a fiber of $\mu$. The relevant moduli space over the local model $\mathbb{C}^{r-1} \times C$ will be further reduced to a suitable moduli space over $\mathbb{C}^{r}$, to which torus actions will be applied. The contribution of $\chi\left(\mathfrak{M}_{(2), n}\right)$ gives rise to the term (1.1.3). 


\section{Chapter 2}

\section{Moduli spaces}

\subsection{Basic definitions and constructions}

The notion of a moduli space is central in various areas of mathematics. Since one of the major tasks of mathematics is to classify, or to put similar objects together, invoking the idea of moduli spaces leads to very important and happy consequences. In the field of algebraic geometry, the notion of moduli spaces has been used in so many areas to help to either investigate those spaces by their own, or to understand the objects themselves that are being modulated by the space. A very basic example of a moduli space is the projective space $\mathbb{P}_{\mathbb{C}}^{2}$ over complex numbers. This space classifies all points in $\mathbb{C}^{3}-\{O\}$, where any two points are in the same equivalence class if there exists a line connects them that passes through the origin. While the geometry of points is relatively simple and well understood, the new resulting moduli space carries a more fascinating structure and reveals more interesting properties. To rigidify the problem and make it more concrete, let us start by defining what a moduli problem is, and then examine what a natural definition of a moduli space should look like.

Consider the category of schemes, denoted by Sch, and the category of sets, de- 
noted by Set, such that for any object $B$ of $\mathbf{S c h}$, we assign a set $\mathbf{S}(B)$ which is a family of objects over $B$, together with what it means for this family of objects to be over $B$. To be more precise, the elements of $\mathbf{S}(B)$ must have some equivalence relation between them which we are free to choose. Once an equivalence relation have been chosen, we define a functor

$$
\text { F : Sch } \rightarrow \text { Set, }
$$

such that

$$
\mathbf{F}(B)=\mathbf{S}(B) / \text { equivalence relation. }
$$

As much as this description is too general, we can restrict this idea in a way that enables us to relate some nice scheme to this moduli problem. Specifically, we want a space, which we will soon call moduli space, to represent the image of the functor in a way that its geometric points have a connection with the image of the functor $\mathbf{F}$. While we can always come up with moduli spaces of various different structures fitting the description in the previous sentence, possibly trivial ones, one problem might arise here is that these spaces could be discursive and uninformative. To be more explicit, it is not always possible to make the moduli problem along with its chosen functor into something that we can gain important properties and new insights from. Of course, it is not hard to construct a moduli problem with its functor that can be turned into a scheme or a vector space in a trivial way. For an example, we might turn an arbitrary moduli problem into a scheme by just declaring that every point in the image of its moduli functor to be an affine scheme. While this would trivially equip our moduli problem with a scheme structure, we will not get any important information neither about the new scheme itself, nor about the objects being represented by the new 
scheme. To avoid this type of triviality, one needs to attempt to control the moduli problem by choosing its functor in a more precise way that enables us to find deeper structures and properties. For this, we introduce the notion of fine moduli space. Before doing so, we need to introduce further definitions.

Definition 2.1. Consider the functors F, G : Sch $\rightarrow$ Set. A natural transformation $\eta: \mathbf{F} \rightarrow \mathbf{G}$ is a family of Set-morphisms $\eta_{B}: \mathbf{F} B \rightarrow \mathbf{G} B$, where $B$ ranges over all schemes in $\mathbf{S c h}$, such that for each morphism $f: B \rightarrow C$ in $\mathbf{S c h}, \eta_{C} \circ \mathbf{F} f=\mathbf{G} f \circ \eta_{B}$, i.e. the following diagram commutes:

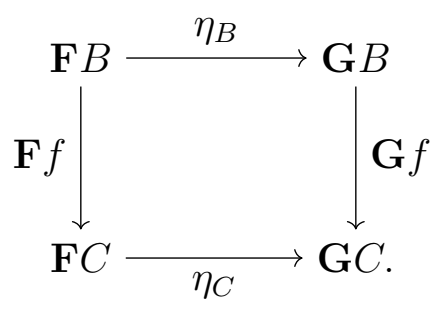

Moreover, if for every object $B$ in $\mathbf{S c h}$, the natural transformation $\eta_{B}$ is an isomorphism in the category Set, then we say that $\eta$ is an isomorphism of functors.

Definition 2.2. Let $\mathfrak{M}$ be an object in the category $\mathbf{S c h}$. Then, we define the functor of points

$$
\operatorname{Mor}_{\mathrm{Sch}}(\ldots, \mathfrak{M}): \operatorname{Sch} \rightarrow \operatorname{Set}
$$

to be the functor that assigns to any scheme $B \in \mathbf{S c h}$, the set of all morphisms $B \rightarrow \mathfrak{M}$

Definition 2.3. The functor $\mathbf{F}$ is representable in the category of schemes if there exist a scheme $\mathfrak{M}$, and a natural transformation $\eta: \mathbf{F} \rightarrow \mathbf{M o r}_{\mathbf{S c h}}(\ldots, \mathfrak{M})$ that is an isomorphism. 
Definition 2.4. Let $\mathbf{F}$ be a moduli functor for the moduli problem $\mathbf{S}(B)$ which is representable by a scheme $\mathfrak{M}$. Then, we say that the scheme $\mathfrak{M}$ is a fine moduli space for the moduli problem $\mathbf{S}(B)$.

A significant outcome of representability is the existence of a univer sal family. The universal family works as a dictionary translating information about the geometry of the families of objects $\mathbf{S}(B)$ and the geometry of the fine moduli space $\mathfrak{M}$ representing it. To make this observation more concrete, let $\mathfrak{C}$ be the family in $\mathbf{S}(\mathfrak{M})$ corresponding to $\operatorname{Id}_{\mathfrak{M}} \in \operatorname{Mor}_{\mathbf{S c h}}(\mathfrak{M}, \mathfrak{M})$. Then, $\mathfrak{C}$ is called the universal family over the fine moduli space $\mathfrak{M}$. The universality of the family $\mathfrak{C}$ is interpreted in the following sense: let $\Psi: \mathfrak{D} \rightarrow B$ be any family in $\mathbf{S}(B)$. Let $\eta$ be as in above, and put $\chi=\eta(\Psi)$. Then there is a commutative fibered product diagram

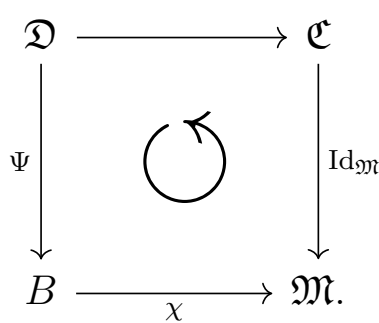

After having introduced the notion of fine moduli spaces, we will examine two examples of some moduli problems: one can be represented by a fine moduli space, but the other can not. In the next section, we will construct in more details the central objects we will be working with throughout this thesis which are the Hilbert scheme and Quot scheme.

Example 2.5. Let $\mathbf{F}$ be the functor of the moduli problem that assigns the set of lines passing through the origin of $\mathbb{A}_{k}^{n+1}$. Then $\mathbb{P}_{k}^{n}$ is the fine moduli space representing that functor, where $k$ is algebraically closed field. 
Example 2.6. In this example, we will show the existence of some moduli problems that can not be represented by a fine moduli space. Before we start the example, which will be a very general phenomenon, let us examine the following situation. Suppose we are trying to find a moduli space that parametrizes all 1-dimensional vector spaces up to isomorphism, then one would expect the moduli space to be just a point. We know a family of 1-dimensional vector spaces over a circle would give a line bundle over the base variety $S^{1}$. Also, it is well known that over a circle, we have two non-isomorphic line bundles, namely, a cylinder and a Mobius strip. Therefore, it is impossible for such a fine moduli space to exist. A more general case would be the possibility of the existence of a moduli space $\mathfrak{M}_{g}$ whose geometric points are the isomorphism classes of smooth curves of genus $g$ over a base scheme $S$, i.e. a scheme $\mathfrak{M}_{g}$ that represents the moduli functor

$$
F: \text { Sch } \rightarrow \text { Set }
$$

that assigns to a scheme $S$, the set of isomorphism classes of smooth curves of genus $g$ over $S$. Again, such a fine moduli space $\mathfrak{M}_{g}$ would be impossible as some of these curves have non-trivial automorphisms.

\subsection{Hilbert scheme and Grothendieck Quot scheme}

\subsubsection{Hilbert scheme}

While it is more natural to construct the Grothendieck Quot scheme first, and then proceed to show how to obtain the Hilbert scheme from it, it is more insightful to start with constructing the Hilbert scheme, and then proceed to the more general Grothendieck Quot scheme. The treatment here is exactly the same as what a reader 
would find in [Qin, Nit]. Following the general discussion in the previous section, we will resolve specific moduli problems in which their functors are representable by schemes called Hilbert schemes and Grothendieck Quot schemes, respectively.

Let $X$ be a quasi projective complex scheme over a locally noetherian scheme $S$, i.e. we have a morphism $\mu: X \rightarrow S$. Let $\mathcal{O}_{X}(1)$ be a very ample line bundle on $X$. Consider the contravarient functor

$$
\mathcal{H} i l b_{X / S}: \text { Sch } \rightarrow \text { Set }
$$

given by

$$
\mathcal{H i l b}_{X / S}(U):\left\{Z \subset U \times{ }_{S} X \mid Z \text { is a closed subscheme of } U \times X \text {, flat over } U\right\} \text {. }
$$

For a morphism $\phi: V \rightarrow U$ of locally noetherian $S$-schemes we have

$$
\mathcal{H} i l b_{X / S}(\phi): \mathcal{H}_{i l b_{X / S}}(U) \rightarrow \mathcal{H} i l b_{X / S}(V)
$$

which sends $Z$ to $Z \times{ }_{U} V$.

Let $q: Z \rightarrow U$ be the projection, and $u \in U$. We put $Z_{u}=q^{*}(u)$. Then, the Hilbert polynomial of $Z$ at $u$ is

$$
p_{u}(m):=\chi\left(\mathcal{O}_{Z_{u}}(m)\right)=\chi\left(\mathcal{O}_{Z_{u}} \otimes_{\mathcal{O}_{X}} \mathcal{O}_{X}(m)\right)
$$

where $p_{u}$ is a polynomial in the variable $m$ independent of the choice of $u$. Let $\mathcal{H} i l b_{X / S}^{p}$ be the subfunctor of $\mathcal{H} i l b_{X / S}$ that associates to $U$ the set of closed subschemes of $X$ which are flat over $U$, and have Hilbert polynomial $p$. Then, we have the following theorem that can be found in [Qin, Nit]: 
Theorem 2.7. the functor $\mathcal{H i l b}_{X / S}^{p}$ is represented by a projective scheme $\mathrm{Hilb}_{X / S}^{p}$, called the Hilbert scheme.

From the discussion right after Definition 2.4, we should be able to describe a universal family that comes with this Hilbert scheme. Specifically, there exists a closed subscheme $\mathcal{Z} \subset \operatorname{Hilb}_{X / S}^{p} \times X$ such that $\mathcal{Z} \rightarrow \operatorname{Hilb}_{X / S}^{p}$ is the universal family in the following sense: if $Z$ is flat family of closed subschemes in $X$ parametrized by $U$ such that $p$ is the Hilbert polynomial of the closed subschemes, then there exists a unique morphism $f: U \rightarrow \operatorname{Hilb}_{X / S}^{p}$ such that

$$
Z=\left(f \times \operatorname{Id}_{X}\right)^{*} \mathcal{Z}
$$

Finally, if $S=\operatorname{Spec} \mathbb{C}$ and the Hilbert polynomial $p(m)=n$ is a constant positive integer, then we drop $S$ from the notation, call $\mathrm{Hilb}_{X}^{p}$ the Hilbert scheme of $n$ points in $X$, and denote it by $X^{[n]}$.

\subsubsection{Quot scheme}

Similarly, I will briefly sketch the construction of Grothendieck Quot scheme. This construction goes exactly as in [Nit]. Let $X \rightarrow S$ be a finite type scheme over a noetherian scheme $S$. Let $E$ be a coherent sheaf on $X$. Let $\mathbf{S c h}_{S}$ be the category of locally noetherian schemes $T$ over $S$. A family of quotients of $E$ parametrized by $T$ is a pair $(\mathcal{F}, q)$ consists of

1. a coherent sheaf $\mathcal{F}$ on $X_{T}=X \times_{S} T$ such that the $S u p p \mathcal{F}$ is proper over $T$ and $\mathcal{F}$ is flat over $T$, together with

2. a surjective $\mathcal{O}_{X_{T}}$-linear homomorphism of sheaves $q: E_{T} \rightarrow \mathcal{F}, E_{T}=\pi^{*}(E)$ where $\pi: X_{T} \rightarrow X$ is the projection. 
Two families $(\mathcal{F}, q)$, and $(\mathcal{G}, f)$ parametrized by $T$ are equivalent if $\operatorname{ker}(q)=\operatorname{ker}(f)$. We denote the equivalence class of $(\mathcal{F}, q)$ by $\langle\mathcal{F}, q\rangle$. The pull-back of $\langle\mathcal{F}, q\rangle$ under an $S$-morphism $T^{\prime} \rightarrow T$ is well defined. Therefore, we have a contravarient functor denoted by $\mathfrak{Q} u o t_{E / X / S}$ from the category $\mathbf{S c h}_{S}$ to the category Set given by

$$
\mathfrak{Q} u o t_{E / X / S}(T)=\{\text { All }<\mathcal{F}, q>\text { parametrized by } T\} \text {. }
$$

If $E=\mathcal{O}_{X}$, then the functor $\mathfrak{Q} u o t_{\mathcal{O}_{X} / X / S}$ associates to $T$ the set of closed subschemes $Y \subset X \times_{S} T$ that are proper and flat over $T$. Namely, this functor is the functor $\mathcal{H}_{i l b_{X / S}}(T)$ defined above. As in the Hilbert scheme case, the Grothendieck Quot scheme is the scheme representing the functor $\mathfrak{Q} u o t_{E / X / S}$, and is denoted by $\operatorname{Quot}_{E / X / S}$.

Let $\mathcal{L}$ be a relatively very ample line bundle on $X$. Let $\mathcal{F}$ be any coherent sheaf on $X$ whose schematic support is proper over $S$ and which is flat over $S$. Let $s \in S$ be a point, $X_{s}$ be the fiber over the point $s$, and $\mathcal{F}_{s}$ be the restriction of $\mathcal{F}$ to $X_{s}$. Then, the Hilbert polynomial is given by

$$
p_{s}(m)=\chi\left(\mathcal{F}_{s}(m)\right)=\chi\left(X_{s}, \mathcal{F}_{s} \otimes \mathcal{L}_{s}^{\otimes m}\right)
$$

where $\mathcal{L}_{s}$ is again the restriction of $\mathcal{L}$ to $X_{s}$. It is important to note as well that we have the following decomposition

$$
\mathfrak{Q} u o t_{E / X / S}=\coprod_{p} \mathfrak{Q} u o t_{E / X / S}^{p, \mathcal{L}} .
$$

We interpret the subfunctor $\mathfrak{Q} u o t_{E / X / S}^{p, \mathcal{L}}$ as follows: for any polynomial $p(m) \in \mathbb{Q}[m]$, 
$\mathfrak{Q u o t}_{E / X / S}^{p, \mathcal{L}}(T)=\left\{\begin{array}{l}\text { all equivalence classes }<\mathcal{F}, q>\text { such that for any } t \in T \\ \text { the Hilbert polynomial of the restriction } \mathcal{F}_{t} \text { computed } \\ \text { with respect to the pull-back of } \mathcal{L} \text { is } p(m) .\end{array}\right\}$ 


\section{Chapter 3}

\section{Virtual Hodge polynomials and Euler characteristics}

Danilov and Khovanskii [DK] introduced virtual Hodge polynomials for reduced complex schemes. These polynomials can be viewed as a convenient tool for computing the Hodge numbers of smooth projective varieties by reducing to computing those of simpler varieties. They can also be used to compute Euler characteristics. In this chapter, we recall the basic properties of virtual Hodge polynomials.

First of all, let $Y$ be a reduced complex scheme (not necessarily projective, irreducible or smooth). Mixed Hodge structures are defined on the cohomology $H_{c}^{k}(Y, \mathbb{Q})$ with compact support (see [Del, DK]). The mixed Hodge structures coincide with the classical one if $Y$ is projective and smooth. For each pair of integers $(m, n)$, define the virtual Hodge number

$$
e^{m, n}(Y)=\sum_{k}(-1)^{k} h^{m, n}\left(H_{c}^{k}(Y, \mathbb{Q})\right) .
$$

Then the virtual Hodge polynomial of $Y$ is defined to be

$$
e(Y ; s, t)=\sum_{m, n} e^{m, n}(Y) s^{m} t^{n}
$$

Next, for an arbitrary complex scheme $Y$, we put

$$
e(Y ; s, t)=e\left(Y_{\text {red }} ; s, t\right)
$$


following [Che]. By (3.0.2) and the results in [DK, Ful, Che] for reduced complex schemes, we see that virtual Hodge polynomials satisfy the following properties:

(i) When $Y$ is projective and smooth, $e(Y ; s, t)$ is the usual Hodge polynomial of $Y$. For a general complex scheme $Y$, we have

$$
e(Y ; 1,1)=\chi(Y)
$$

where $\chi(Y)$ denotes the topological Euler number of $Y$.

(ii) If $Y=\coprod_{i=1}^{n} Y_{i}$ is a finite disjoint union of locally closed subsets, then

$$
e(Y ; s, t)=\sum_{i=1}^{n} e\left(Y_{i} ; s, t\right)
$$

(iii) If $f: Y \rightarrow Y^{\prime}$ is a Zariski-locally trivial bundle with fiber $F$, then

$$
e(Y ; s, t)=e\left(Y^{\prime} ; s, t\right) \cdot e(F ; s, t)
$$

(iv) If $f: Y \rightarrow Y^{\prime}$ is a bijective morphism, then

$$
e(Y ; s, t)=e\left(Y^{\prime} ; s, t\right)
$$

By the Lemma 5.6 in [Che] and the Theorem 4.1 in [LY], if $Y$ is a reduced complex scheme with a $\mathbb{C}^{*}$-action and if $Y^{\mathbb{C}^{*}}$ denotes the set of fixed points, then

$$
\chi(Y)=\chi\left(Y^{\mathbb{C}^{*}}\right)
$$




\section{Chapter 4}

\section{Torus actions on $\operatorname{Hilb}^{n}\left(\mathbb{C}^{r}, O\right)$}

\section{1 $\quad r$-dimensional partitions}

In this section we introduce torus action following [LQ, Che]. This technique allows us to reduce computations of $\mathfrak{M}_{\lambda, n}$ (see Definition 5.9) to simpler computations of moduli spaces $\mathfrak{M}_{\xi, n ; n_{0}}^{\mathbb{C}^{r-1} \times C}$ (see Definition 6.4), which will be further reduced to computations of the punctual moduli spaces $\mathfrak{M}_{\xi, n ; L, O}^{\mathbb{C}^{r}}$ (see chapters 6.1, and 6.2).

\section{Definition 4.1.}

(i) Let $r \geq 2$ and $n \geq 0$. An $r$-dimensional partition of $n$ is an array

$$
\left(n_{i_{1}, \ldots, i_{r-1}}\right)_{i_{1}, \ldots, i_{r-1}}
$$

of nonnegative integers $n_{i_{1}, \ldots, i_{r-1}}$ indexed by the tuples

$$
\left(i_{1}, \ldots, i_{r-1}\right) \in\left(\mathbb{Z}_{\geq 0}\right)^{r-1}
$$

such that

$$
\sum_{i_{1}, \ldots, i_{r-1}} n_{i_{1}, \ldots, i_{r-1}}=n
$$

and $n_{i_{1}, \ldots, i_{r-1}} \geq n_{j_{1}, \ldots, j_{r-1}}$ whenever $i_{1} \leq j_{1}, \ldots, i_{r-1} \leq j_{r-1}$. 
(ii) Let $\mathfrak{I}$ be a finite subset of $\left(\mathbb{Z}_{\geq 0}\right)^{r-1}$. Then an $\mathfrak{I}$-punctual $r$-dimensional partitions of $n$ is an array

$$
\left(n_{i_{1}, \ldots, i_{r-1}}\right)_{i_{1}, \ldots, i_{r-1}}
$$

of nonnegative integers $n_{i_{1}, \ldots, i_{r-1}}$ indexed by the tuples

$$
\left(i_{1}, \ldots, i_{r-1}\right) \in\left(\mathbb{Z}_{\geq 0}\right)^{r-1}-\mathfrak{I}
$$

such that

$$
\sum_{i_{1}, \ldots, i_{r-1}} n_{i_{1}, \ldots, i_{r-1}}=n
$$

and $n_{i_{1}, \ldots, i_{r-1}} \geq n_{j_{1}, \ldots, j_{r-1}}$ whenever $i_{1} \leq j_{1}, \ldots, i_{r-1} \leq j_{r-1}$.

(iii) Define $P_{r}^{\Im}(n)$ to be the number of $\mathfrak{I}$-punctual $r$-dimensional partitions of $n$. For simplicity, we denote $P_{r}^{\Im}(n)$ by $P_{r}(n), \widetilde{P}_{r}(n), A_{r}(n), B_{r}(n)$ when

$$
\mathfrak{I}=\emptyset,\{(0, \ldots, 0)\},\{(0, \ldots, 0),(1,0, \ldots 0)\},\{(0, \ldots, 0),(1,0, \ldots, 0),(0, \ldots, 0,1)\},
$$

respectively.

We remark that Definition 4.1 (i) is consistent with the one used in [MNOP1], while our $r$-dimensional partitions are $(r-1)$-dimensional partitions in [Che].

\subsection{Generating series of plane partitions and torus action}

Lemma 4.2. The series $\sum_{n=0}^{+\infty} P_{r}^{\Im}(n) q^{n}$ is convergent if $|q|<1 / r$.

Proof. It suffices to prove that

$$
P_{r}^{\mathfrak{I}}(n) \leqslant r \cdot P_{r}^{\mathfrak{I}}(n-1) .
$$


Let $S_{r}^{\mathfrak{\Im}}(n)$ be the set of $\mathfrak{I}$-punctual $r$-dimensional partitions of $n$. Let

$$
\lambda=\left(n_{i_{1}, \ldots, i_{r-1}}\right)_{i_{1}, \ldots, i_{r-1}} \in S_{r}^{\mathfrak{J}}(n) .
$$

Define

$$
\begin{aligned}
j_{1} & =\max \left\{i_{1} \mid n_{i_{1}, \ldots, i_{r-1}}>0\right\}, \\
j_{2} & =\max \left\{i_{2} \mid n_{j_{1}, i_{2}, \ldots, i_{r-1}}>0\right\}, \\
\vdots & \\
j_{r-1} & =\max \left\{i_{r-1} \mid n_{j_{1}, \ldots, j_{r-2}, i_{r-1}}>0\right\} .
\end{aligned}
$$

By definition, $n_{j_{1}, \ldots, j_{r-1}}>0$. Define an element

$$
f(\lambda)=\left(m_{i_{1}, \ldots, i_{r-1}}\right)_{i_{1}, \ldots, i_{r-1}} \in S_{r}^{\mathfrak{I}}(n-1)
$$

by putting:

$$
m_{i_{1}, \ldots, i_{r-1}}= \begin{cases}n_{i_{1}, \ldots, i_{r-1}}-1, & \text { if }\left(i_{1}, \ldots, i_{r-1}\right)=\left(j_{1}, \ldots, j_{r-1}\right) \\ n_{i_{1}, \ldots, i_{r-1}}, & \text { otherwise. }\end{cases}
$$

This defines a map

$$
f: S_{r}^{\mathfrak{I}}(n) \rightarrow S_{r}^{\mathfrak{I}}(n-1) .
$$

Note that $f$ is surjective. Moreover, $\left|f^{-1}(\mu)\right| \leqslant r$ for each $\mu \in S_{r}^{\Im}(n-1)$. Therefore, we obtain (4.2.1).

Torus actions on the punctual Hilbert scheme $\operatorname{Hilb}^{n}\left(\mathbb{C}^{r}, O\right)$ have been studied in [LQ, Che]. Let $z_{1}, \ldots, z_{r}$ be the coordinate functions of $\mathbb{C}^{r}$. Then $\mathbb{C}^{*}$ acts on $\mathbb{C}^{r}$ by

$$
t\left(z_{1}, \ldots, z_{r}\right)=\left(t^{w_{1}} z_{1}, \ldots, t^{w_{r}} z_{r}\right), \quad t \in \mathbb{C}^{*}
$$


This $\mathbb{C}^{*}$-action on $\mathbb{C}^{r}$ induces a $\mathbb{C}^{*}$-action on $\operatorname{Hilb}^{n}\left(\mathbb{C}^{r}, O\right)$. Now choose $w_{1}, \ldots, w_{r} \in \mathbb{Z}$ properly. Then, the $\mathbb{C}^{*}$-fixed points in $\operatorname{Hilb}^{n}\left(\mathbb{C}^{r}, O\right)$ are precisely those corresponding to the colength- $n$ ideals of $\mathbb{C}\left[z_{1}, \ldots, z_{r}\right]$ generated by monomials. These ideals are in one-to-one correspondence with $r$-dimensional partitions of $n$. Indeed, given an $r$-dimensional partition $\left(n_{i_{1}, \ldots, i_{r-1}}\right)_{i_{1}, \ldots, i_{r-1} \geq 0}$ of $n$, the ideal of $\mathbb{C}\left[z_{1}, \ldots, z_{r}\right]$ generated by the monomials $z_{1}^{i_{1}} \cdots z_{r-1}^{i_{r-1}} z_{r}^{n_{i_{1}, \ldots, i_{r-1}}}$ has colength- $n$. Conversely, given a colength- $n$ ideal $I$ of $\mathbb{C}\left[z_{1}, \ldots, z_{r}\right]$ generated by monomials, we obtain an $r$-dimensional partition $\left(n_{i_{1}, \ldots, i_{r-1}}\right)_{i_{1}, \ldots, i_{r-1} \geq 0}$ of $n$ by putting

$$
n_{i_{1}, \ldots, i_{r-1}}=\min \left\{i_{r} \mid z_{1}^{i_{1}} \cdots z_{r-1}^{i_{r-1}} z_{r}^{i_{r}} \in I\right\} .
$$

Therefore, by (3.0.7), we have (see the Proposition 5.1 in [Che]):

$$
\chi\left(\operatorname{Hilb}^{n}\left(\mathbb{C}^{r}, O\right)\right)=P_{r}(n) .
$$

Proposition 4.3. We have

$$
\begin{aligned}
\sum_{n=0}^{+\infty} P_{2}(n) q^{n} & =\prod_{n=1}^{+\infty} \frac{1}{1-q^{n}} \\
\sum_{n=0}^{+\infty} P_{3}(n) q^{n} & =\prod_{n=1}^{+\infty} \frac{1}{\left(1-q^{n}\right)^{n}} \\
\sum_{n=0}^{+\infty} \tilde{P}_{3}(n) q^{n} & =\frac{1}{1-q} \sum_{n=0}^{+\infty} P_{3}(n) q^{n} .
\end{aligned}
$$

Proof. Formula (4.2.5) follows from the definition of $P_{2}(n)$. Formula (4.2.6) is known as MacMahon's formula. The formula and its proof can be found in [And]. Formula (4.2.7) was proved in [LQ].

\subsection{Important relation}

In the following Proposition we prove an important relation that would be used later. 
Proposition 4.4. Let $|q|<1 / 3$. Then

$$
\sum_{n=0}^{+\infty} A_{3}(n) q^{n}=\frac{1}{1-q} \cdot \frac{1}{1-q^{2}} \cdot \sum_{n=0}^{+\infty} P_{3}(n) q^{n}
$$

Proof. We shall use notations and results from Sect. 11.2 of [And]. Identify our 3dimensional partitions with the plane partitions there, i.e., our 3-dimensional partition $\left(n_{i_{1}, i_{2}}\right)_{i_{1}, i_{2} \geq 0}$ is identified with the plane partition whose entry at the lattice point $\left(i_{1}, i_{2}\right), i_{1}, i_{2} \geq 0$ in the plane is equal to $n_{i_{1}, i_{2}}$. Similarly, our punctual 3 -dimensional partitions will correspond to the punctual plane partitions.

Let $S_{k, \ell}(m, n), \widetilde{S}_{k, \ell}(m, n), \bar{S}_{k, \ell}(m, n), \hat{S}_{k, \ell}(m, n)$ denote the sets of $\mathfrak{I}$-punctual plane partitions of $m$ with

$$
\mathfrak{I}=\emptyset,\{(0,0)\},\{(0,0),(1,0)\},\{(0,0),(1,0),(0,1)\}
$$

respectively, and with at most $\ell$ columns, at most $k$ rows, and with each entry $\leq n$.

Let $p_{k, \ell}(m, n)=\left|S_{k, \ell}(m, n)\right|, \tilde{p}_{k, \ell}(m, n)=\left|\widetilde{S}_{k, \ell}(m, n)\right|, \bar{p}_{k, \ell}(m, n)=\left|\bar{S}_{k, \ell}(m, n)\right|$, and $\hat{p}_{k, \ell}(m, n)=\left|\hat{S}_{k, \ell}(m, n)\right|$. Define the generating functions:

$$
\begin{aligned}
& \pi_{k, \ell}(n ; q)=\sum_{m=0}^{+\infty} p_{k, \ell}(m, n) q^{m}, \\
& \tilde{\pi}_{k, \ell}(n ; q)=\sum_{m=0}^{+\infty} \tilde{p}_{k, \ell}(m, n) q^{m}, \\
& \bar{\pi}_{k, \ell}(n ; q)=\sum_{m=0}^{+\infty} \bar{p}_{k, \ell}(m, n) q^{m}, \\
& \hat{\pi}_{k, \ell}(n ; q)=\sum_{m=0}^{+\infty} \hat{p}_{k, \ell}(m, n) q^{m} .
\end{aligned}
$$

So

$$
\pi_{+\infty,+\infty}(+\infty ; q)=\sum_{m=0}^{+\infty} P_{3}(m) q^{m}
$$




$$
\bar{\pi}_{+\infty,+\infty}(+\infty ; q)=\sum_{m=0}^{+\infty} A_{3}(m) q^{m}
$$

Define

$$
(q)_{i}=(1-q)\left(1-q^{2}\right) \cdots\left(1-q^{i}\right)
$$

for a positive integer $i$. By the Theorem 11.2 in [And],

$$
\pi_{k, \ell}(n ; q)=\frac{(q)_{1}(q)_{2} \cdots(q)_{k-1}}{(q)_{\ell}(q)_{\ell+1} \cdots(q)_{\ell+k-1}} \cdot \frac{(q)_{n+\ell}(q)_{n+\ell+1} \cdots(q)_{n+\ell+k-1}}{(q)_{n}(q)_{n+1} \cdots(q)_{n+k-1}} .
$$

By (7.14) in [LQ], we have

$$
\tilde{\pi}_{k, \ell}(n ; q)=q^{-n}\left[\pi_{k, \ell}(n ; q)-\pi_{k, \ell}(n-1 ; q)\right] .
$$

There is a relation among the numbers of different punctual plane partitions:

$$
\tilde{p}_{k, l}(m, n)=\tilde{p}_{k, l}(m, n-1)+2 \bar{p}_{k, l}(m-n, n)-\hat{p}_{k, l}(m-2 n, n)
$$

which can be illustrated by the following diagram

$$
\begin{aligned}
& {\left[\begin{array}{cccc}
\vdots & \vdots & \vdots & \\
* & * & * & \cdots \\
* & * & * & \cdots \\
& * & * & \cdots
\end{array}\right]} \\
& =\left[\begin{array}{cccc}
\vdots & \vdots & \vdots & \\
* & * & * & \cdots \\
* & * & * & \cdots \\
& * & * & \cdots
\end{array}\right]+\left[\begin{array}{cccc}
\vdots & \vdots & \vdots & \\
* & * & * & \cdots \\
* & * & * & \cdots \\
& n & * & \ldots
\end{array}\right]+\left[\begin{array}{cccc}
\vdots & \vdots & \vdots & \\
* & * & * & \ldots \\
n & * & * & \ldots \\
& * & * & \ldots
\end{array}\right]-\left[\begin{array}{cccc}
\vdots & \vdots & \vdots & \\
* & * & * & \ldots \\
n & * & * & \ldots \\
n & * & \ldots
\end{array}\right] . \\
& \text { with parts } \leqslant \text { n-1 } \quad \text { with parts } \leqslant n \quad \text { with parts } \leqslant n \quad \text { with parts } \leqslant \text { n }
\end{aligned}
$$

In other words, every punctual plane partition with empty $(0,0)$-entry and parts $\leqslant n$ is either a punctual plane partition with empty $(0,0)$-entry and parts $\leqslant(n-1)$, or a punctual plane partition with empty $(0,0)$-entry, $n$ at $(1,0)$-entry and parts $\leqslant n$, or 
a punctual plane partition with empty $(0,0)$-entry, $n$ at $(0,1)$-entry and parts $\leqslant n$. Therefore

$$
\begin{aligned}
& \tilde{\pi}_{k, \ell}(n ; q) \\
= & \tilde{\pi}_{k, \ell}(n-1 ; q)+2 \sum_{m=0}^{\infty} \bar{p}_{k, l}(m-n, n) q^{m}-\sum_{m=0}^{\infty} \hat{p}_{k, l}(m-2 n, n) q^{m} \\
= & \tilde{\pi}_{k, \ell}(n-1 ; q)+2 q^{n} \cdot \sum_{m=0}^{\infty} \bar{p}_{k, l}(m-n, n) q^{m-n}-q^{2 n} \cdot \sum_{m=0}^{\infty} \hat{p}_{k, l}(m-2 n, n) q^{m-2 n} \\
= & \tilde{\pi}_{k, \ell}(n-1 ; q)+2 q^{n} \cdot \bar{\pi}_{k, \ell}(n ; q)-q^{2 n} \cdot \hat{\pi}_{k, \ell}(n ; q) .
\end{aligned}
$$

So

$$
2 q^{n} \cdot \bar{\pi}_{k, \ell}(n ; q)-q^{2 n} \cdot \hat{\pi}_{k, \ell}(n ; q)=\tilde{\pi}_{k, \ell}(n ; q)-\tilde{\pi}_{k, \ell}(n-1 ; q)
$$

Now, we want to use a parallel argument to the one found in [LQ]. To take the limits $k, \ell, n \rightarrow+\infty$, we assume $|q|<1 / 3$ in the rest of the proof. By (4.3.4), (4.3.5) and the definition of $(q)_{i}$ from (4.3.3), we have

$$
\begin{aligned}
& \bar{\pi}_{k, \ell}(n ; q)-\frac{q^{n}}{2} \cdot \hat{\pi}_{k, \ell}(n ; q) \\
= & \frac{q^{-2 n}}{2} \cdot\left[\pi_{k, \ell}(n ; q)-\pi_{k, \ell}(n-1 ; q)\right]-\frac{q^{-(2 n-1)}}{2} \cdot\left[\pi_{k, \ell}(n-1 ; q)-\pi_{k, \ell}(n-2 ; q)\right] \\
= & \frac{\pi_{k, \ell}(n-2 ; q)}{2} \cdot \frac{q^{-2 n} \cdot I_{k, \ell}(n ; q)}{\prod_{i=0}^{k-1}\left(1-q^{n+i-1}\right) \cdot \prod_{i=0}^{k-1}\left(1-q^{n+i}\right)}
\end{aligned}
$$

where $I_{k, \ell}(n ; q)$ is defined to be

$$
\begin{aligned}
& q \cdot\left(1-q^{n-1}\right)\left(1-q^{n}\right)^{2} \cdots\left(1-q^{n+k-2}\right)^{2} \cdot\left(1-q^{n+k-1}\right) \\
- & (1+q) \cdot \prod_{i=0}^{k-1}\left(1-q^{n+\ell+i-1}\right) \cdot \prod_{i=0}^{k-1}\left(1-q^{n+i}\right) \\
+ & \left(1-q^{n+\ell-1}\right)\left(1-q^{n+\ell}\right)^{2} \cdots\left(1-q^{n+\ell+k-2}\right)^{2} \cdot\left(1-q^{n+\ell+k-1}\right) .
\end{aligned}
$$

It is straightforward to check that $I_{k, \ell}(n ; q)$ is equal to

$$
q \cdot\left(q^{n-1}\left(2 q^{n}+2 q^{n+1}+\cdots+2 q^{n+k-2}+q^{n+k-1}\right)\right.
$$




$$
\begin{aligned}
& +q^{n}\left(q^{n}+2 q^{n+1}+\cdots+2 q^{n+k-2}+q^{n+k-1}\right) \\
& +q^{n}\left(2 q^{n+1}+\cdots+2 q^{n+k-2}+q^{n+k-1}\right) \\
& +\cdots \\
& +q^{n+k-3}\left(q^{n+k-3}+2 q^{n+k-2}+q^{n+k-1}\right) \\
& +q^{n+k-3}\left(2 q^{n+k-2}+q^{n+k-1}\right) \\
& +q^{n+k-2}\left(q^{n+k-2}+q^{n+k-1}\right) \\
& \left.+q^{n+k-2} \cdot q^{n+k-1}\right) \\
& -\quad(1+q) \cdot\left(q^{n} \cdot\left(q^{n+1}+\cdots+q^{n+k-1}\right)\right. \\
& \left.+q^{n+1} \cdot\left(q^{n+2}+\cdots+q^{n+k-1}\right)+\cdots+q^{n+k-2} \cdot q^{n+k-1}\right) \\
& +\quad q^{2 n+\ell-1} \cdot f_{k, \ell}(n, q)+q^{3 n} \cdot f_{k}(n, q)
\end{aligned}
$$

where $\left|f_{k}(n, q)\right|<g_{k}(q)$ and $\left|f_{k, \ell}(n, q)\right|<h_{k}(q)$ for some functions $g_{k}(q)$ and $h_{k}(q)$ of $k$ and $q$. Therefore,

$$
\begin{aligned}
& \lim _{k \rightarrow+\infty} \lim _{n, \ell \rightarrow+\infty} \frac{q^{-2 n} I_{k, n}(n ; q)}{2} \\
= & 1+q+2 q^{2}+2 q^{3}+3 q^{4}+3 q^{5}+\ldots \\
= & (1+q) \cdot\left(1+2 q^{2}+3 q^{4}+4 q^{6}+\cdots\right) \\
= & (1+q) \cdot \frac{1}{\left(1-q^{2}\right)^{2}} \\
= & \frac{1}{1-q} \cdot \frac{1}{1-q^{2}} .
\end{aligned}
$$

Combining this with (4.3.6) and Lemma 4.2, we obtain

$$
\bar{\pi}_{+\infty,+\infty}(+\infty ; q)=\pi_{+\infty,+\infty}(+\infty ; q) \cdot \frac{1}{1-q} \cdot \frac{1}{1-q^{2}}
$$


By (4.3.1) and (4.3.2),

$$
\sum_{n=0}^{+\infty} A_{3}(n) q^{n}=\frac{1}{1-q} \cdot \frac{1}{1-q^{2}} \cdot \sum_{n=0}^{+\infty} P_{3}(n) q^{n}
$$

Conjecture 4.5. Let $|q|<1 / 3, \ell \geqslant 0$ and $\mathfrak{I}_{\ell}=\{(0,0), \cdots,(0, \ell)\}$. Then,

$$
\sum_{n=0}^{+\infty} P_{3}^{\mathfrak{I}_{\ell}}(n) q^{n}=\prod_{i=1}^{\ell+1} \frac{1}{1-q^{i}} \cdot \sum_{n=0}^{+\infty} P_{3}(n) q^{n}
$$

By (4.2.7) and Proposition 4.4, Conjecture 4.5 holds for $\ell=0,1$. 


\section{Chapter 5}

\section{The moduli spaces $\mathfrak{I}_{n}(X, \beta)$ and $\mathfrak{M}_{d, n}$}

\section{$5.1 \quad$ Description of $\mathfrak{I}_{n}(X, \beta)$ and $\mathfrak{M}_{d, n}$}

Let $X$ be a smooth projective complex variety of dimension $r$. For a fixed class $\beta \in H_{2}(X ; \mathbb{Z})$ and a fixed integer $n$, following the definitions and notations in [LQ], we define $\mathfrak{I}_{n}(X, \beta)$ to be the moduli space of 1 -dimensional closed subschemes $Z$ of $X$ satisfying the two conditions:

$$
\chi\left(\mathcal{O}_{Z}\right)=n, \quad[Z]=\beta
$$

where $[Z]$ is the class associated to the dimension-1 component (weighted by their intrinsic multiplicities) of $Z$.

Lemma 5.1. If $\operatorname{dim}(X)=3$, and $H$ is a divisor on $X$, then

$$
\chi\left(\mathcal{O}_{Z}(d H)\right)=([Z] \cdot H) \cdot d+\chi\left(\mathcal{O}_{Z}\right)=(\beta \cdot H) \cdot d+n .
$$

Proof. By the Hirzebruch-Riemann-Roch formula, we have

$$
\chi\left(\mathcal{O}_{Z}(d H)\right)=\chi\left(\mathcal{O}_{Z} \otimes \mathcal{O}_{X}(d H)\right)
$$




$$
\begin{aligned}
= & \left(\operatorname{ch}\left(\mathcal{O}_{Z} \otimes \mathcal{O}_{X}(d H)\right) \cdot \operatorname{td}\left(T_{X}\right)\right)_{3} \\
= & \left(\operatorname{ch}\left(\mathcal{O}_{Z}\right) \cdot \operatorname{ch}\left(\mathcal{O}_{X}(d H)\right) \cdot \operatorname{td}\left(T_{X}\right)\right)_{3} \\
= & \left(\left(0+0-c_{2}\left(\mathcal{O}_{Z}\right)+\frac{1}{2} c_{3}\left(\mathcal{O}_{Z}\right)\right)\right. \\
& \left.\cdot\left(1+d H+\frac{1}{2} d^{2} H+\frac{1}{6} d^{3} H^{3}\right) \cdot \operatorname{td}\left(T_{X}\right)\right)_{3} \\
= & \left(\left(-c_{2}\left(\mathcal{O}_{Z}\right)+\frac{1}{2} c_{3}\left(\mathcal{O}_{Z}\right)-d H \cdot c_{2}\left(\mathcal{O}_{Z}\right)\right) \cdot \operatorname{td}\left(T_{X}\right)\right)_{3} \\
= & d H \cdot[Z]+\chi\left(\mathcal{O}_{Z}\right)
\end{aligned}
$$

since $c_{2}\left(\mathcal{O}_{Z}\right)=-[Z]=-\beta$.

The degree-0 moduli space $\mathfrak{I}_{n}(X, 0)$ is isomorphic to the Hilbert scheme $X^{[n]}$ parametrizing length- $n$ 0-dimensional closed subschemes of $X$. In general, when $\beta \neq 0$ and when an ample divisor $H$ on $X$ is fixed, the space $\mathfrak{I}_{n}(X, \beta)$ is only part of the Hilbert scheme (see [Gro]) defined in terms of certain degree-1 Hilbert polynomial. By Lemma 5.1, when $\operatorname{dim}(X)=3$, this degree-1 Hilbert polynomial is $(\beta \cdot H) \cdot d+n$. By the Lemma 1 in [MNOP2], when $\operatorname{dim}(X)=3$, the virtual dimension of $\mathfrak{I}_{n}(X, \beta)$ is

$$
-\left(\beta \cdot K_{X}\right)
$$

In the rest of the paper, we adopt the following basic assumptions.

Assumption 5.2. We assume that $X$ admits a Zariski-locally trivial fibration

$$
\mu: X \rightarrow S
$$

where $S$ is a smooth projective variety, the fibers are smooth irreducible curves of genus- $g$, and $\beta \in H_{2}(X ; \mathbb{Z})$ is the class of a fiber of $\mu$. 
Let $d, n \geq 0$. Then,

$$
\mathfrak{I}_{d(1-g)}(X, d \beta) \cong S^{[d]}
$$

where $S^{[d]}$ denotes the Hilbert scheme of $d$ points on the variety $S$. Put

$$
\mathfrak{M}_{d, n}=\mathfrak{I}_{d(1-g)+n}(X, d \beta)
$$

An element in $\mathfrak{M}_{d, n}$ denotes a 1-dimensional closed subscheme $Z$ of $X$ whose 1dimensional component is equal to some curve $C_{\xi} \in \mathfrak{M}_{d, 0}$ (i.e, $I_{Z} \subset I_{C_{\xi}}$ ) such that the quotient $I_{C_{\xi}} / I_{Z}$ is supported at finitely many points in $X$ with

$$
h^{0}\left(X, I_{C_{\xi}} / I_{Z}\right)=n
$$

Our goal is to determine, under Assumption 5.2, the partition function for the Euler characteristics of the moduli spaces $\mathfrak{M}_{d, n}=\mathfrak{I}_{d(1-g)+n}(X, d \beta), n \geq 0$ :

$$
\sum_{n=0}^{+\infty} \chi\left(\mathfrak{M}_{d, n}\right) q^{n}
$$

Lemma 5.3. Let $\mathcal{Z}_{d} \subset S^{[d]} \times S$ be the universal codimension-2 subscheme, and $\operatorname{Quot}_{\left(\operatorname{Id}_{S}[d] \times \mu\right)^{*} I_{\mathcal{Z}_{d}} / S^{[d]} \times X / S^{[d]}}^{n}$ be the Grothendieck Quot-scheme with the constant polynomial $n$. Then, there exists an isomorphism

$$
\left.\mathfrak{M}_{d, n} \cong \operatorname{Quot}_{\left(\operatorname{Id}_{S}[d]\right.}^{n} \times \mu\right)^{*} I_{\mathcal{Z}_{d}} / S[d] \times X / S^{[d]} .
$$

Proof. Note that every element in $\mathfrak{M}_{d, n}=\mathfrak{I}_{d(1-g)+n}(X, d \beta)$ is of the form:

$$
Z=\Xi+\Theta
$$

where $\Xi \in X^{\left[n-n_{0}\right]}$ for some $n_{0}$ satisfying $0 \leq n_{0} \leq n, \operatorname{Supp}(\Xi) \cap \operatorname{Supp}(\Theta)=\emptyset$, and the dimension-1 component $\Theta$ is equal to some curves $C_{\xi} \in \mathfrak{M}_{d, 0}$ together with 
embedded points of length- $n_{0}$ (i.e., $I_{\Theta} \subset I_{C_{\xi}}$ and the quotient $I_{C_{\xi}} / I_{\Theta}$ is supported at finitely many points in $C_{\xi}$ with $\left.h^{0}\left(X, I_{C_{\xi}} / I_{\Theta}\right)=n_{0}\right)$. So we have a surjection

$$
I_{C_{\xi}} \rightarrow I_{C_{\xi}} / I_{Z} \rightarrow 0
$$

where the quotient $I_{C_{\xi}} / I_{Z}$ is supported at finitely many points, and has length $n$.

It follows that the universal quotient over $\operatorname{Quot}_{\left(\operatorname{Id}_{S^{[d]}} \times \mu\right)^{*} I_{\mathcal{Z}_{d}} / S^{[d]} \times X / S^{[d]}}^{n}$ induces a bijective morphism $\phi_{1}: \operatorname{Quot}_{\left(\mathrm{Id}_{S^{[d]}} \times \mu\right)^{*} I_{Z_{d}} / S^{[d]} \times X / S^{[d]}}^{n} \rightarrow \mathfrak{M}_{d, n}$.

On the other hand, let $\mathcal{I}_{d, n}$ be the universal ideal sheaf over $\mathfrak{M}_{d, n} \times X$. Let $\mathcal{I}_{d, n}^{\prime}$ be the saturation of $\mathcal{I}_{d, n} \subset \mathcal{O}_{\mathfrak{M}_{d, n} \times X}$ (see Definition 1.1.5 in [HL]). Then, $\mathcal{I}_{d, n}^{\prime}$ is a flat family of ideal sheaves in $\mathfrak{M}_{d, 0} \cong S^{[d]}$, and fits in an exact sequence

$$
0 \rightarrow \mathcal{I}_{d, n} \rightarrow \mathcal{I}_{d, n}^{\prime} \rightarrow \mathcal{Q} \rightarrow 0
$$

over $\mathfrak{M}_{d, n} \times X$. Now the flat family $\mathcal{I}_{d, n}^{\prime}$ and the quotient $\mathcal{I}_{d, n}^{\prime} \rightarrow \mathcal{Q} \rightarrow 0$ induces a morphism $\phi_{2}: \mathfrak{M}_{d, n} \rightarrow \operatorname{Quot}_{\left(\mathrm{Id}_{S^{[d]}} \times \mu\right)^{*} I_{Z_{d}} / S[d] \times X / S^{[d]}}^{n}$ which is inverse to $\phi_{1}$.

In view of Lemma 5.3, we will make no difference between $\mathfrak{M}_{d, n}$ and the Quotscheme Quot $\left.\left.\operatorname{(Id}_{S^{[d]}}^{n} \times \mu\right)^{*} I_{Z_{d}} / S^{[d]} \times X / S^{[d]}\right]$. In particular, we have a natural morphism:

$$
\mathfrak{M}_{d, n} \rightarrow S^{[d]}
$$

Moreover, over $\mathfrak{M}_{d, n} \times X$, there exists a universal quotient

$$
p_{d, n}^{*} I_{\mathcal{Z}_{d}} \rightarrow \mathcal{Q} \rightarrow 0
$$

where $p_{d, n}$ is the composition of the morphism $\mathfrak{M}_{d, n} \times X \rightarrow S^{[d]} \times X$ induced from (5.1.9) and the morphism $\operatorname{Id}_{S^{[d]}} \times \mu: S^{[d]} \times X \rightarrow S^{[d]} \times S$.

Definition 5.4. Let $\beta$ and $g$ be from Assumption 5.2. Let $0 \leq n_{0} \leq n$. 
(i) We define $\mathfrak{M}_{d, n ; n_{0}}$ to be the locally closed subset of $\mathfrak{M}_{d, n}$ consisting of all the elements $Z=\Xi+\Theta$ from (5.1.8) such that $h^{0}\left(X, I_{C_{\xi}} / I_{\Theta}\right)=n_{0}$.

(ii) Let $C$ be a union of fibers of $X \rightarrow S$. Fix a point $x \in C$. We define $\mathfrak{M}_{d, n ; C}$ (respectively, $\mathfrak{M}_{d, n ; C, x}$ ) to be the closed subset of $\mathfrak{M}_{d, n ; n}$ consisting of all the elements $Z=\Xi+\Theta$ from (5.1.8) such that $\Xi=\emptyset$, and $\operatorname{Supp}(\Theta)=C$ (respectively, the embedded points in $\Theta$ are supported at $x$ ).

(iii) Let $C$ be a union of fibers of $X \rightarrow S$. Fix a point $x \in C$. Define $X_{C}^{[n], n_{0}}$ to be the locally closed subset of $X^{[n]}$ consisting of all the elements $\Xi=\Xi_{1}+\Xi_{2} \in X^{[n]}$ such that $\operatorname{Supp}\left(\Xi_{1}\right) \cap C=\emptyset, \operatorname{Supp}\left(\Xi_{2}\right) \subset C$, and $\ell\left(\Xi_{2}\right)=n_{0}$. Define $X_{C}^{[n]}=$ $X_{C}^{[n], n}$, and define $X_{x}^{[n]}$ to be the closed subset of $X^{[n]}$ consisting of all $\Xi \in X^{[n]}$ such that $\operatorname{Supp}(\Xi)=\{x\}$ (i.e., $X_{x}^{[n]}$ is the punctual Hilbert scheme at $x$ ).

Remark 5.5. To emphasis the dependence on $X$, we will also denote the notations $\mathfrak{M}_{d, n}, \mathfrak{M}_{d, n ; n_{0}}, \mathfrak{M}_{d, n ; C}, \ldots$ by $\mathfrak{M}_{d, n}^{X}, \mathfrak{M}_{d, n ; n_{0}}^{X}, \mathfrak{M}_{d, n ; C}^{X}, \ldots$ respectively

Let $S^{(d)}$ be the $d$-th symmetric product of $S$.

Definition 5.6. For $0 \leq n_{0} \leq n$, define $Z_{d, n ; n_{0}}$ to be the locally closed subset of $X^{[n]} \times S^{(d)}$ consisting of all pairs $(\Xi, \xi)$ such that

$$
\Xi=\Xi_{1}+\Xi_{2}
$$

$\operatorname{Supp}\left(\Xi_{1}\right) \cap \mu^{-1}(\operatorname{Supp}(\xi))=\emptyset, \operatorname{Supp}\left(\Xi_{2}\right) \subset \mu^{-1}(\operatorname{Supp}(\xi))$, and $\ell\left(\Xi_{1}\right)=n_{0}$. Put

$$
W_{d, n}=Z_{d, n ; n} \text { and } T_{d, n}=Z_{d, n ; 0} .
$$

Lemma 5.7. Let $0 \leq n_{0} \leq n$. Then there exists a bijective morphism:

$$
W_{d, n-n_{0}} \times_{S^{(d)}} \mathfrak{M}_{d, n_{0} ; n_{0}} \rightarrow \mathfrak{M}_{d, n ; n_{0}}
$$


Proof. Let $\pi_{1}$ and $\pi_{2}$ be the two projections of $W_{d, n-n_{0}} \times_{S^{(d)}} \mathfrak{M}_{d, n_{0} ; n_{0}}$.

Over $\mathfrak{M}_{d, n_{0} ; n_{0}} \times X$, there exists a universal quotient

$$
p_{d, n_{0}}^{*} I_{\mathcal{Z}_{d}} \rightarrow \mathcal{Q}_{1} \rightarrow 0 .
$$

So over $\left(W_{d, n-n_{0}} \times_{S^{(d)}} \mathfrak{M}_{d, n_{0} ; n_{0}}\right) \times X$, we have a surjection:

$$
\left(\pi_{2} \times \mathrm{Id}_{X}\right)^{*} p_{d, n_{0}}^{*} I_{\mathcal{Z}_{d}} \rightarrow\left(\pi_{2} \times \mathrm{Id}_{X}\right)^{*} \mathcal{Q}_{1} \rightarrow 0
$$

Also, over $X^{\left[n-n_{0}\right]} \times X$, we have a universal quotient:

$$
\mathcal{O}_{X^{\left[n-n_{0}\right]} \times X} \rightarrow \mathcal{Q}_{2} \rightarrow 0
$$

Hence, over $\left(W_{d, n-n_{0}} \times_{S^{(d)}} \mathfrak{M}_{d, n_{0} ; n_{0}}\right) \times X$, we have another surjection:

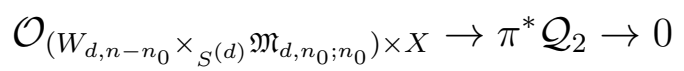

where $\pi$ is the composition:

$$
\left(W_{d, n-n_{0}} \times_{S^{(d)}} \mathfrak{M}_{d, n_{0} ; n_{0}}\right) \times X \rightarrow W_{d, n-n_{0}} \times X \rightarrow X^{\left[n-n_{0}\right]} \times X .
$$

Note:

$$
\begin{gathered}
\operatorname{Supp}\left(\left(\pi_{2} \times \operatorname{Id}_{X}\right)^{*} \mathcal{Q}_{1}\right) \subset\left(\pi_{2} \times \operatorname{Id}_{X}\right)^{-1} p_{d, n_{0}}^{-1}\left(\mathcal{Z}_{d}\right), \\
\left(\pi_{2} \times \operatorname{Id}_{X}\right)^{-1} p_{d, n_{0}}^{-1}\left(\mathcal{Z}_{d}\right) \cap \operatorname{Supp}\left(\pi^{*} \mathcal{Q}_{2}\right)=\emptyset
\end{gathered}
$$

So we get a surjection:

$$
\left(\pi_{2} \times \operatorname{Id}_{X}\right)^{*} p_{d, n_{0}}^{*} I_{\mathcal{Z}_{d}} \rightarrow\left(\pi_{2} \times \operatorname{Id}_{X}\right)^{*} \mathcal{Q}_{1} \oplus \pi^{*} \mathcal{Q}_{2} \rightarrow 0,
$$

and the quotient is flat over $W_{d, n-n_{0}} \times_{S^{(d)}} \mathfrak{M}_{d, n_{0} ; n_{0}}$. This surjection induces a morphism 


$$
\Psi: W_{d, n-n_{0}} \times_{S(d)} \mathfrak{M}_{d, n_{0} ; n_{0}} \rightarrow \mathfrak{M}_{d, n}
$$

One checks that $\operatorname{im}(\Psi)=\mathfrak{M}_{d, n ; n_{0}}$ and $\Psi$ is injective.

Remark 5.8. Similarly, there exists a bijective morphism:

$$
W_{d, n_{0}} \times_{S^{(d)}} T_{d, n-n_{0}} \rightarrow Z_{d, n ; n_{0}} .
$$

Let $\lambda=\left(\lambda_{1} \geq \ldots \geq \lambda_{\ell}\right)$ be a partition of $d$. Let $S^{(\lambda)}$ be the locally closed subset of the $d$-th symmetric product $S^{(d)}$ consisting of all the elements of the form

$$
\xi=\lambda_{1} s_{1}+\ldots+\lambda_{\ell} s_{\ell}
$$

where $s_{1}, \ldots, s_{\ell}$ are distinct points in $S$.

Definition 5.9. Let $\lambda \vdash d$ be a partition of $d$. We define $\mathfrak{M}_{\lambda, n}$ to be the locally closed subset of $\mathfrak{M}_{d, n}$ consisting of all the elements $Z=\Xi+\Theta$ in (5.1.8) such that the support of $\Theta$ is equal to $\mu^{*}(\xi)$ for some $\xi \in S^{(\lambda)}$. We adopt similar notations $\mathfrak{M}_{\lambda, n ; n_{0}}, \mathfrak{M}_{\lambda, n ; C}, \mathfrak{M}_{\lambda, n ; C, x}, Z_{\lambda, n ; n_{0}}, W_{\lambda, n}, T_{\lambda, n}$ as those in Definition 5.4 and Definition 5.6.

By Definition 5.9, we have the following decompositions:

$$
\begin{aligned}
\mathfrak{M}_{d, n} & =\coprod_{\lambda \vdash d} \mathfrak{M}_{\lambda, n}, \\
\mathfrak{M}_{\lambda, n} & =\coprod_{n_{0}=0}^{n} \mathfrak{M}_{\lambda, n ; n_{0}} .
\end{aligned}
$$

Moreover, as in Lemma 5.7 and Remark 5.8, we have the following bijective morphisms:

$$
W_{\lambda, n-n_{0}} \times_{S(\lambda)} \mathfrak{M}_{\lambda, n_{0} ; n_{0}} \rightarrow \mathfrak{M}_{\lambda, n ; n_{0}}
$$




$$
W_{\lambda, n_{0}} \times_{S^{(\lambda)}} T_{\lambda, n-n_{0}} \rightarrow Z_{\lambda, n ; n_{0}}
$$

Let $d=2$. Then, there are exactly two partitions of $d=2:(2),\left(1^{2}\right)$. So by $(5.1 .20)$ and (5.1.21), we have

$$
\begin{aligned}
\mathfrak{M}_{2, n} & =\mathfrak{M}_{(2), n} \coprod \mathfrak{M}_{\left(1^{2}\right), n}, \\
\mathfrak{M}_{\left(1^{2}\right), n} & =\coprod_{n_{0}=0}^{n} \mathfrak{M}_{\left(1^{2}\right), n, n_{0}}, \\
\mathfrak{M}_{(2), n} & =\coprod_{n_{0}=0}^{n} \mathfrak{M}_{(2), n, n_{0}} .
\end{aligned}
$$

\section{$5.2 \quad$ Various bijective morphisms}

Now we fix some notations. Let $C$ denote a fixed fiber of the fibration $\mu: X \rightarrow S$. Let $m=\operatorname{dim}(X)-1=r-1, O$ be the origin of $\mathbb{C}^{m}$, and $C_{0}=\{O\} \times C$.

The following is Proposition 4.3 in [LQ].

Proposition 5.10. Let $O$ be the origin of $\mathbb{C}^{m}$ and $C_{0}=\{O\} \times C$. Let $f: U \rightarrow \mathbb{C}^{m}$ be an étale morphism. Then there exists a bijective morphism over $U$ :

$$
\Psi_{f}: \mathfrak{M}_{1, n ; C_{0}}^{\mathbb{C}^{m} \times C} \times U \rightarrow \mathfrak{M}_{1, n ; n}^{U \times C}
$$

The goal of this section is to generalize Proposition 5.10 to the case when $\lambda=(2)$.

Lemma 5.11. There exists a bijective morphism over $\mathbb{C}^{m}$ :

$$
\Psi: \mathfrak{M}_{(2), n ; C_{0}}^{\mathbb{C}^{m} \times C} \times \mathbb{C}^{m} \rightarrow \mathfrak{M}_{(2), n ; n}^{\mathbb{C}^{m} \times C}
$$

Proof. Let $X_{0}=\mathbb{C}^{m} \times C$. We view $X_{0}$ as a fibration over $\mathbb{C}^{m}$ with the projection $X_{0}=\mathbb{C}^{m} \times C \rightarrow \mathbb{C}^{m}$. Over $\mathfrak{M}_{(2), n ; C_{0}}^{X_{0}} \times X_{0}$, there exists a universal quotient

$$
\mathcal{F}_{0} \rightarrow \mathcal{Q}_{0} \rightarrow 0
$$


where $\mathcal{F}_{0}$ is the pull-back of the universal sheaf coming from the punctual Hilbert scheme $\operatorname{Hilb}^{2}\left(\mathbb{C}^{m}, O\right) \times \mathbb{C}^{m}$. Let

$$
\sigma: \mathbb{C}^{m} \times \mathbb{C}^{m} \rightarrow \mathbb{C}^{m}
$$

be the subtraction: $\sigma(u, v)=u-v$. Let $\Sigma=\operatorname{Id}_{\mathfrak{M}_{(2), n ; C_{0}}^{X_{0}}} \times \sigma \times \operatorname{Id}_{C}$ :

$\mathfrak{M}_{(2), n ; C_{0}}^{X_{0}} \times \mathbb{C}^{m} \times X_{0}=\mathfrak{M}_{(2), n ; C_{0}}^{X_{0}} \times \mathbb{C}^{m} \times \mathbb{C}^{m} \times C \rightarrow \mathfrak{M}_{(2), n ; C_{0}}^{X_{0}} \times \mathbb{C}^{m} \times C=\mathfrak{M}_{(2), n ; C_{0}}^{X_{0}} \times X_{0}$

Then we obtain a commutative diagram of morphisms:

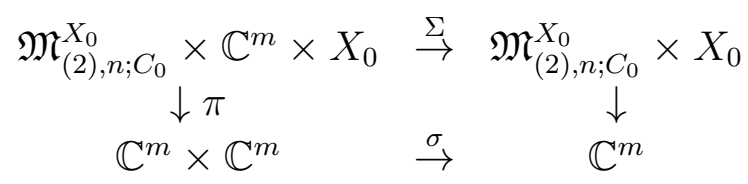

where the two vertical morphisms are the natural projections. We have over $\mathfrak{M}_{(2), n ; C_{0}}^{X_{0}} \times$ $\mathbb{C}^{m} \times X_{0}$ a quotient

$$
\Sigma^{*} \mathcal{F}_{0} \rightarrow \Sigma^{*} \mathcal{Q}_{0} \rightarrow 0
$$

Let $u \in \mathbb{C}^{m}$. The restriction of (5.2.2) to $\mathfrak{M}_{(2), n ; C_{0}}^{X_{0}} \times\{u\} \times X_{0} \cong \mathfrak{M}_{(2), n ; C_{0}}^{X_{0}} \times X_{0}$ is

$$
\Sigma_{u}^{*} \mathcal{F}_{0} \rightarrow \Sigma_{u}^{*} \mathcal{Q}_{0} \rightarrow 0
$$

where $\Sigma_{u}$ is the automorphism of $\mathfrak{M}_{(2), n ; C_{0}}^{X_{0}} \times X_{0}=\mathfrak{M}_{(2), n ; C_{0}}^{X_{0}} \times \mathbb{C}^{m} \times C$ induced by

$$
\sigma_{u}: \mathbb{C}^{m} \rightarrow \mathbb{C}^{m}
$$

with $\sigma_{u}(v)=u-v$. By the universal property, (5.2.2) induces a morphism:

$$
\Psi: \mathfrak{M}_{(2), n ; C_{0}}^{X_{0}} \times \mathbb{C}^{m} \rightarrow \mathfrak{M}_{(2), n ; n}^{X_{0}}
$$

The morphism $\Psi$ is bijective since every $\Sigma_{u}$ is an automorphism. 
Let $U$ be a smooth variety. Let

$$
M_{2}(U)=\left\{\xi \in(U)^{[2]} \mid \xi \text { is supported at one point }\right\} .
$$

Since $U \cong U^{((2))}$, the natural morphism $\mathfrak{M}_{(2), n ; n}^{U \times C} \rightarrow U^{((2))} \subset U^{(2)}$ induces the mor$\operatorname{phism} \mathfrak{M}_{(2), n ; n}^{U \times C} \rightarrow U$.

Lemma 5.12. Let $f: U \rightarrow \mathbb{C}^{m}$ be an étale morphism. Then there exists a bijective morphism $\widetilde{\Psi}_{f}: \mathfrak{M}_{(2), n ; n}^{\mathbb{C}^{m} \times C} \times_{\mathbb{C}^{m}} U \rightarrow \mathfrak{M}_{(2), n ; n}^{U \times C}$ over $U$.

Proof. Let $X=U \times C$ and $X_{0}=\mathbb{C}^{m} \times C$. Then there exists a universal quotient

$$
\left(\pi_{0}\right)^{*} I_{\mathcal{Z}_{2}\left(\mathbb{C}^{m}\right)} \rightarrow \mathcal{Q} \rightarrow 0
$$

over $\mathfrak{M}_{(2), n ; n}^{X_{0}} \times X_{0}$, where $\mathcal{Z}_{2}\left(\mathbb{C}^{m}\right)$ is the universal subscheme of $M_{2}\left(\mathbb{C}^{m}\right) \times \mathbb{C}^{m}$ and $\pi_{0}$ is the composition:

$$
\mathfrak{M}_{(2), n ; n}^{X_{0}} \times X_{0} \rightarrow M_{2}\left(\mathbb{C}^{m}\right) \times X_{0}=M_{2}\left(\mathbb{C}^{m}\right) \times\left(\mathbb{C}^{m} \times C\right) \rightarrow M_{2}\left(\mathbb{C}^{m}\right) \times \mathbb{C}^{m}
$$

The projection $\mathfrak{M}_{(2), n ; n}^{X_{0}} \times_{\mathbb{C}^{m}} U \rightarrow \mathfrak{M}_{(2), n ; n}^{X_{0}}$ and the morphism $f \times \operatorname{Id}_{C}: X \rightarrow X_{0}$ induce

$$
F:\left(\mathfrak{M}_{(2), n ; n}^{X_{0}} \times_{\mathbb{C}^{m}} U\right) \times X \rightarrow \mathfrak{M}_{(2), n ; n}^{X_{0}} \times X_{0}
$$

Moreover it is known that there exists a bijective morphism over $U$ :

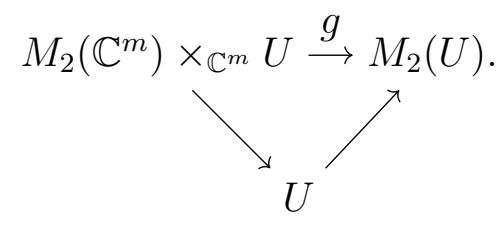

Let $\tilde{f}$ be the follwing morphism which is induced by $f$ :

$$
\left(M_{2}\left(\mathbb{C}^{m}\right) \times_{\mathbb{C}^{m}} U\right) \times U \longrightarrow\left(M_{2}\left(\mathbb{C}^{m}\right) \times_{\mathbb{C}^{m}} \mathbb{C}^{m}\right) \times \mathbb{C}^{m}=M_{2}\left(\mathbb{C}^{m}\right) \times \mathbb{C}^{m}
$$


We have a commutative diagram:

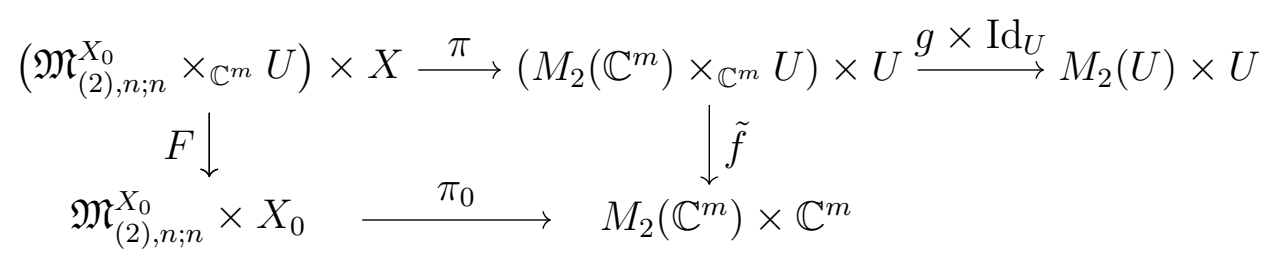

Pulling-back the surjection (5.2.3) via $F$, we obtain the surjection

$$
F^{*}\left(\pi_{0}\right)^{*} I_{\mathcal{Z}_{2}\left(\mathbb{C}^{m}\right)} \rightarrow F^{*} \mathcal{Q} \rightarrow 0
$$

over $\left(\mathfrak{M}_{(2), n ; n}^{X_{0}} \times \mathbb{C}_{\mathbb{C}^{m}} U\right) \times X$. Since $F^{*}\left(\pi_{0}\right)^{*}=\left(\pi_{0} \circ F\right)^{*}=(\tilde{f} \circ \pi)^{*}=\pi^{*} \tilde{f}^{*}$, we have the surjection:

$$
\pi^{*} \tilde{f}^{*} I_{\mathcal{Z}_{2}\left(\mathbb{C}^{m}\right)} \rightarrow F^{*} \mathcal{Q} \rightarrow 0
$$

over $\left(\mathfrak{M}_{(2), n ; n}^{X_{0}} \times_{\mathbb{C}^{m}} U\right) \times X$. Note that

$$
\begin{aligned}
& \left(g \times \operatorname{Id}_{U}\right)^{-1}\left(\mathcal{Z}_{2}(U)\right)=\left\{(\xi, u ; u) \in\left(M_{2}\left(\mathbb{C}^{m}\right) \times_{\mathbb{C}^{m}} U\right) \times U \mid \operatorname{Supp}(\xi)=f(u)\right\}, \\
& \tilde{f}^{-1}\left(\mathcal{Z}_{2}\left(\mathbb{C}^{m}\right)\right)=\left\{\left(\xi, u_{1} ; u_{2}\right) \in\left(M_{2}\left(\mathbb{C}^{m}\right) \times_{\mathbb{C}^{m}} U\right) \times U \mid \operatorname{Supp}(\xi)=f\left(u_{1}\right)=f\left(u_{2}\right)\right\} .
\end{aligned}
$$

Since $f: U \rightarrow \mathbb{C}^{m}$ is étale, we conclude that $\tilde{f}^{-1}\left(\mathcal{Z}_{2}\left(\mathbb{C}^{m}\right)\right)$ is the disjoint union of $\left(g \times \operatorname{Id}_{U}\right)^{-1}\left(\mathcal{Z}_{2}(U)\right)$ and some other irreducible components. Hence,

$$
\begin{aligned}
\left.\pi^{*} \tilde{f}^{*} I_{\mathcal{Z}_{2}\left(\mathbb{C}^{m}\right)}\right|_{\pi^{-1}\left(g \times \operatorname{Id}_{U}\right)^{-1}\left(\mathcal{Z}_{2}(U)\right)} & =\left.\pi^{*} I_{\left(g \times \operatorname{Id}_{U}\right)^{-1}\left(\mathcal{Z}_{2}(U)\right)}\right|_{\pi^{-1}\left(g \times \operatorname{Id}_{U}\right)^{-1}\left(\mathcal{Z}_{2}(U)\right)} \\
& =\left.\tilde{\pi}^{*} I_{\mathcal{Z}_{2}(U)}\right|_{\tilde{\pi}^{-1}\left(\mathcal{Z}_{2}(U)\right)}
\end{aligned}
$$

where $\tilde{\pi}=\left(g \times \operatorname{Id}_{U}\right) \circ \pi$. So we obtain a surjection over $\left(\mathfrak{M}_{(2), n ; n}^{X_{0}} \times_{\mathbb{C}^{m}} U\right) \times X$ :

$$
\left.\left.\tilde{\pi}^{*} I_{\mathcal{Z}_{2}(U)} \rightarrow \tilde{\pi}^{*} I_{\mathcal{Z}_{2}(U)}\right|_{\tilde{\pi}^{-1}\left(\mathcal{Z}_{2}(U)\right)} \rightarrow F^{*} \mathcal{Q}\right|_{\tilde{\pi}^{-1}\left(\mathcal{Z}_{2}(U)\right)} \rightarrow 0 .
$$

One checks that $\left.F^{*} \mathcal{Q}\right|_{\tilde{\pi}^{-1}\left(\mathcal{Z}_{2}(U)\right)}$ is flat over $\mathfrak{M}_{(2), n ; n}^{X_{0}} \times_{\mathbb{C}^{m}} U$ and that the quotient (5.2.7) induces a morphism:

$$
\widetilde{\Psi}_{f}: \mathfrak{M}_{(2), n ; n}^{X_{0}} \times_{\mathbb{C}^{m}} U \rightarrow \mathfrak{M}_{(2), n ; n}^{X}
$$


over $U$. Using the completions of the points in $U$, we see that $\widetilde{\Psi}_{f}$ is bijective.

Parallel to Proposition 5.10 we have

Proposition 5.13. Let $O$ be the origin of $\mathbb{C}^{m}$ and $C_{0}=\{O\} \times C$. Let $f: U \rightarrow \mathbb{C}^{m}$ be an étale morphism. Then there exists a bijective morphism over $U$ :

$$
\Psi_{f}: \mathfrak{M}_{(2), n ; C_{0}}^{\mathbb{C}^{m} \times C} \times U \rightarrow \mathfrak{M}_{(2), n ; n}^{U \times C}
$$

Proof. Follows from Lemmas 5.11 and 5.12 by putting $\Psi_{f}=\widetilde{\Psi}_{f} \circ\left(\Psi \times_{\mathbb{C}^{m}} \operatorname{Id}_{U}\right)$. 


\section{Chapter 6}

\section{Reducing the problem and the computation of $\chi\left(\mathfrak{M}_{(2), n}\right)$}

\subsection{Reduction to the local model $\mathbb{C}^{r-1} \times C$}

Lemma 6.1. Let $O$ be the origin of $\mathbb{C}^{r-1}$ and $C_{0}=\{O\} \times C$. Then,

$$
\sum_{n=0}^{+\infty} e\left(\mathfrak{M}_{(2), n} ; s, t\right) q^{n}=\sum_{n=0}^{+\infty} e\left(W_{(2), n} ; s, t\right) q^{n} \cdot \sum_{n=0}^{+\infty} e\left(\mathfrak{M}_{(2), n ; C_{0}}^{\mathbb{C}^{r-1} \times C} ; s, t\right) q^{n} .
$$

Proof. By (5.1.26), (5.1.22), (3.0.4) and (3.0.6), we obtain:

$$
e\left(\mathfrak{M}_{(2), n} ; s, t\right)=\sum_{n_{0}=0}^{n} e\left(\mathfrak{M}_{(2), n ; n_{0}} ; s, t\right)=\sum_{n_{0}=0}^{n} e\left(W_{(2), n-n_{0}} \times_{S((2))} \mathfrak{M}_{(2), n_{0} ; n_{0}} ; s, t\right) .
$$

Since $S^{((2))} \cong S$, we have

$$
e\left(\mathfrak{M}_{(2), n} ; s, t\right)=\sum_{n_{0}=0}^{n} e\left(W_{(2), n-n_{0}} \times_{S} \mathfrak{M}_{(2), n_{0} ; n_{0}} ; s, t\right) .
$$

Consider the commutative diagram for the fiber product $W_{(2), n-n_{0}} \times_{S} \mathfrak{M}_{(2), n_{0} ; n_{0}}$ :

$$
\begin{array}{ccc}
W_{(2), n-n_{0}} \times_{S} \mathfrak{M}_{(2), n_{0} ; n_{0}} & \longrightarrow & \mathfrak{M}_{(2), n_{0} ; n_{0}} \\
\downarrow & & \downarrow \phi_{1} \\
W_{(2), n-n_{0}} & \longrightarrow & S .
\end{array}
$$

By the Proposition I.3.24 in [Mil], there exist an open affine cover $\left\{U_{i}\right\}_{i}$ of $S$ and étale morphisms $f_{i}: U_{i} \rightarrow \mathbb{C}^{r-1}$. By Proposition 5.13, we see that for each $i$, there exists a bijective morphism over the open affine subset $U_{i}$ :

$$
\Psi_{f_{i}}: \mathfrak{M}_{(2), n_{0} ; C_{0}}^{\mathbb{C}^{r-1} \times C} \times U_{i} \rightarrow\left(\phi_{2}\right)^{-1}\left(U_{i}\right)
$$


So there exist a decomposition $S=\coprod_{i} S_{i}$ of locally closed subsets $S_{i}$ and bijective morphisms $\Psi_{S_{i}}: \mathfrak{M}_{(2), n_{0} ; C_{0}}^{\mathbb{C}^{r-1} \times C} \times S_{i} \rightarrow\left(\phi_{2}\right)^{-1}\left(S_{i}\right)$. By (6.1.3), there exist a decomposition

$$
W_{(2), n-n_{0}}=\coprod_{i} W_{(2), n-n_{0} ; i}
$$

of locally closed subsets $W_{(2), n-n_{0} ; i}$ and bijective morphisms

$$
\Psi_{W_{(2), n-n_{0} ; i}}: \mathfrak{M}_{(2), n_{0} ; C_{0}}^{\mathbb{C}^{r-1} \times C} \times W_{(2), n-n_{0} ; i} \rightarrow\left(\phi_{1}\right)^{-1}\left(W_{(2), n-n_{0} ; i}\right)
$$

Combining this with (3.0.4) and (3.0.6), we conclude that

$$
\begin{aligned}
e\left(W_{(2), n-n_{0}} \times_{S} \mathfrak{M}_{(2), n_{0} ; n_{0}} ; s, t\right) & =\sum_{i} e\left(\left(\phi_{1}\right)^{-1}\left(W_{(2), n-n_{0} ; i}\right) ; s, t\right) \\
& =\sum_{i} e\left(\mathfrak{M}_{(2), n_{0} ; C_{0}}^{\mathbb{C}^{r-1} \times C} \times W_{(2), n-n_{0} ; i} ; s, t\right) \\
& =\sum_{i} e\left(W_{(2), n-n_{0} ; i} ; s, t\right) \cdot e\left(\mathfrak{M}_{(2), n_{0} ; C_{0}}^{\mathbb{C}^{r-1} \times C} ; s, t\right) \\
& =e\left(W_{(2), n-n_{0}} ; s, t\right) \cdot e\left(\mathfrak{M}_{(2), n_{0} ; C_{0}}^{\mathbb{C}^{r-1} \times C} ; s\right) .
\end{aligned}
$$

By (6.1.2) and (6.1.4),

$$
\begin{aligned}
\sum_{n=0}^{+\infty} e\left(\mathfrak{M}_{(2), n} ; s, t\right) q^{n} & =\sum_{n=0}^{+\infty} \sum_{n_{0}=0}^{n} e\left(W_{(2), n-n_{0}} ; s, t\right) q^{n-n_{0}} \cdot e\left(\mathfrak{M}_{(2), n_{0} ; C_{0}}^{\mathbb{C}^{r-1} \times C} ; s, t\right) q^{n_{0}} \\
& =\sum_{n=0}^{+\infty} e\left(W_{(2), n} ; s, t\right) q^{n} \cdot \sum_{n=0}^{+\infty} e\left(\mathfrak{M}_{(2), n ; C_{0}}^{\mathbb{C}^{r-1} \times C} ; s, t\right) q^{n}
\end{aligned}
$$

Lemma 6.2. Let $O$ be the origin of $\mathbb{C}^{r-1}$ and $C_{0}=\{O\} \times C$. Then,

$$
\sum_{n=0}^{+\infty} e\left(X^{[n]} \times S ; s, t\right) q^{n}=\sum_{n=0}^{+\infty} e\left(W_{(2), n} ; s, t\right) q^{n} \cdot \sum_{n=0}^{+\infty} e\left(\left(\mathbb{C}^{r-1} \times C\right)_{C_{0}}^{[n]} ; s, t\right) q^{n}
$$

Proof. By Definition 5.9,

$$
X^{[n]} \times S^{(\lambda)}=\coprod_{n_{0}=0}^{n} Z_{\lambda, n ; n_{0}}
$$


So

$$
e\left(X^{[n]} \times S ; s, t\right)=e\left(X^{[n]} \times S^{((2))} ; s, t\right)=\sum_{n_{0}=0}^{n} e\left(Z_{(2), n ; n_{0}} ; s, t\right) .
$$

By (5.1.23), we have an analogue of (6.1.2):

$$
e\left(X^{[n]} \times S ; s, t\right)=\sum_{n_{0}=0}^{n} e\left(W_{(2), n-n_{0}} \times_{S} T_{(2), n_{0}} ; s, t\right) .
$$

Let $\lambda$ be a partition of $n_{0}$, denoted by $\lambda \vdash n_{0}$. Express $\lambda$ as $\lambda=\left(\lambda_{1}, \ldots, \lambda_{\ell}\right)$ where $\lambda_{1} \geq \ldots \geq \lambda_{\ell}$ and $\lambda_{1}+\ldots+\lambda_{\ell}=n_{0}$. We define $T_{(2), \lambda}$ to be the locally closed subset of $T_{(2), n_{0}}$ consisting of all the pairs $(\Xi, s)$ such that $\Xi=\Xi_{1}+\ldots+\Xi_{\ell}$ where

$$
\operatorname{Supp}\left(\Xi_{i}\right)=\left\{x_{i}\right\} \subset \mu^{-1}(s)
$$

$\ell\left(\Xi_{i}\right)=\lambda_{i}$, and the points $x_{1}, \ldots, x_{\ell}$ are distinct. Then,

$$
e\left(X^{[n]} \times S ; s, t\right)=\sum_{n_{0}=0}^{n} \sum_{\lambda \vdash n_{0}} e\left(W_{(2), n-n_{0}} \times_{S} T_{(2), \lambda} ; s, t\right) .
$$

Using the Lemma 2.1.4 in [Go2], we can prove that the natural morphism $T_{(2), \lambda} \rightarrow S$ is a Zariski-locally trivial fibration with fibers isomorphic to $\left(\mathbb{C}^{r-1} \times C\right)_{C_{0}}^{\lambda}$. Here $\left(\mathbb{C}^{r-1} \times C\right)_{C_{0}}^{\lambda}$ denotes the locally closed subset of $\left(\mathbb{C}^{r-1} \times C\right)_{C_{0}}^{\left[n_{0}\right]}$ consisting of

$$
\Xi^{\prime}=\Xi_{1}^{\prime}+\ldots+\Xi_{\ell}^{\prime}
$$

where $\operatorname{Supp}\left(\Xi_{i}^{\prime}\right)=\left\{x_{i}^{\prime}\right\} \subset C_{0}, \ell\left(\Xi_{i}^{\prime}\right)=\lambda_{i}$, and $x_{1}^{\prime}, \ldots, x_{\ell}^{\prime}$ are distinct. Hence

$$
\begin{aligned}
e\left(X^{[n]} \times S ; s, t\right) & =\sum_{n_{0}=0}^{n} \sum_{\lambda \vdash n_{0}} e\left(W_{(2), n-n_{0}} ; s, t\right) \cdot e\left(\left(\mathbb{C}^{r-1} \times C\right)_{C_{0}}^{\lambda} ; s, t\right) \\
& =\sum_{n_{0}=0}^{n} e\left(W_{(2), n-n_{0}} ; s, t\right) \cdot \sum_{\lambda \vdash n_{0}} e\left(\left(\mathbb{C}^{r-1} \times C\right)_{C_{0}}^{\lambda} ; s, t\right) \\
& =\sum_{n_{0}=0}^{n} e\left(W_{(2), n-n_{0}} ; s, t\right) \cdot e\left(\left(\mathbb{C}^{r-1} \times C\right)_{C_{0}}^{\left[n_{0}\right]} ; s, t\right),
\end{aligned}
$$


where we used the fact that $\left(\mathbb{C}^{r-1} \times C\right)_{C_{0}}^{\left[n_{0}\right]}$ is the disjoint union of the locally closed subsets $\left(\mathbb{C}^{r-1} \times C\right)_{C_{0}}^{\lambda}, \lambda \vdash n_{0}$. Therefore,

$$
\begin{aligned}
\sum_{n=0}^{+\infty} e\left(X^{[n]} \times S ; s, t\right) q^{n} & =\sum_{n=0}^{+\infty} \sum_{n_{0}=0}^{n} e\left(W_{(2), n-n_{0}}\right) q^{n_{0}-n} \cdot e\left(\left(\mathbb{C}^{r-1} \times C\right)_{C_{0}}^{\left[n_{0}\right]} ; s, t\right) q^{n_{0}} \\
& =\sum_{n=0}^{+\infty} e\left(W_{(2), n} ; s, t\right) q^{n} \cdot \sum_{n=0}^{+\infty} e\left(\left(\mathbb{C}^{r-1} \times C\right)_{C_{0}}^{[n]} ; s, t\right) q^{n}
\end{aligned}
$$

Proposition 6.3. Let $O$ be the origin of $\mathbb{C}^{r-1}$ and $C_{0}=\{O\} \times C$. Then,

$$
\sum_{n=0}^{+\infty} e\left(\mathfrak{M}_{(2), n} ; s, t\right) q^{n}=\sum_{n=0}^{+\infty} e\left(X^{[n]} ; s, t\right) q^{n} \cdot e(S ; s, t) \cdot \frac{\sum_{n=0}^{+\infty} e\left(\mathfrak{M}_{(2), n ; C_{0}}^{\mathbb{C}^{r-1} \times C} ; s\right) q^{n}}{\sum_{n=0}^{+\infty} e\left(\left(\mathbb{C}^{r-1} \times C\right)_{C_{0}}^{[n]} ; s, t\right) q^{n}}
$$

Proof. By (6.1), we have

$$
\sum_{n=0}^{+\infty} e\left(\mathfrak{M}_{(2), n} ; s, t\right) q^{n}=\sum_{n=0}^{+\infty} e\left(W_{(2), n} ; s, t\right) q^{n} \cdot \sum_{n=0}^{+\infty} e\left(\mathfrak{M}_{(2), n ; C_{0}}^{\mathbb{C}^{r-1} \times C} ; s, t\right) q^{n}
$$

By Lemma 6.2 and (2.5) we have

$$
\begin{aligned}
\sum_{n=0}^{+\infty} e\left(W_{(2), n} ; s, t\right) q^{n} & =\frac{\sum_{n=0}^{+\infty} e\left(X^{[n]} \times S ; s, t\right) q^{n}}{\sum_{n=0}^{+\infty} e\left(\left(\mathbb{C}^{r-1} \times C\right)_{C_{0}}^{[n]} ; s, t\right) q^{n}} \\
e\left(X^{[n]} \times S ; s, t\right) & =e\left(X^{[n]} ; s, t\right) \cdot e(S ; s, t) .
\end{aligned}
$$

By combining $(6,11),(6,12)$ with $(6,10)$ the result follows.

Following Chapter 4 , choose a $\mathbb{C}^{*}$-action on $\mathbb{C}^{r-1}$ such that $\left(\mathbb{C}^{r-1}\right)^{\mathbb{C}^{*}}=\{O\}$ and the induced $\mathbb{C}^{*}$-action on the punctual Hilbert scheme $\operatorname{Hilb}^{2}\left(\mathbb{C}^{r-1}, O\right)$ has finitely many fixed points. Let $z_{1}, \ldots, z_{r-1}$ be the coordinates of $\mathbb{C}^{r-1}$. Then the elements in $\left(\operatorname{Hilb}^{2}\left(\mathbb{C}^{r-1}, O\right)\right)^{\mathbb{C}^{*}}$ are in one-to-one correspondence with the following ideals:

$$
\left(z_{1}^{2}, z_{2}, \ldots, z_{r-2},, z_{r-1}\right), \ldots,\left(z_{1}, z_{2}, \ldots, z_{r-2}, z_{r-1}^{2}\right)
$$


Let $\mathbb{C}^{*}$ act on $\mathbb{C}^{r-1} \times C$ by acting on $C$ trivially. Then $\mathbb{C}^{*}$-action induces an action on the moduli space $\mathfrak{M}_{(2), n ; C_{0}}^{\mathbb{C}^{r-1} \times C}$. Now, if $\Theta \in\left(\mathfrak{M}_{(2), n ; C_{0}}^{\mathbb{C}^{r-1} \times C}\right)^{\mathbb{C}^{*}}$, then the 1-dimensional component of $\Theta$ is equal to $\mu^{*} \xi$ for some $\xi \in\left(\operatorname{Hilb}^{2}\left(\mathbb{C}^{r-1}, O\right)\right)^{\mathbb{C}^{*}}$.

Definition 6.4. For each $\xi \in\left(\operatorname{Hilb}^{2}\left(\mathbb{C}^{r-1}, O\right)\right)^{\mathbb{C}^{*}}$, define

$$
\mathfrak{M}_{\xi, n ; C_{0}}^{\mathbb{C}^{r-1} \times C}=\left\{\Theta \in \mathfrak{M}_{(2), n ; C_{0}}^{\mathbb{C}^{r-1} \times C} \mid \text { the 1-dimensional component of } \Theta \text { is equal to } \mu^{*} \xi\right\} .
$$

Then, each $\mathfrak{M}_{\xi, n ; C_{0}}^{\mathbb{C}^{r-1} \times C}$ admits a $\mathbb{C}^{*}$-action. Moreover,

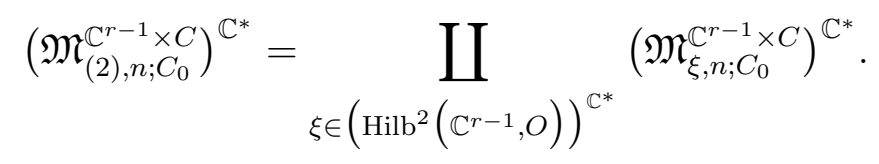

By (3.0.7),

$$
\begin{aligned}
& \chi\left(\mathfrak{M}_{(2), n ; C_{0}}^{\mathbb{C}^{r-1} \times C}\right)=\chi\left(\left(\mathfrak{M}_{(2), n ; C_{0}}^{\mathbb{C}^{r-1} \times C}\right)^{\mathbb{C}^{*}}\right) \\
& =\sum_{\xi \in\left(\mathrm{Hilb}^{2}\left(\mathbb{C}^{r-1}, O\right)\right)} \chi\left(\left(\mathfrak{M}_{\xi, n ; C_{0}}^{\mathbb{C}^{r-1} \times C}\right)^{\mathbb{C}^{*}}\right) \\
& =\sum_{\xi \in\left(\mathrm{Hilb}^{2}\left(\mathbb{C}^{r-1}, O\right)\right)} \chi\left(\mathfrak{M}_{\xi, n ; C_{0}^{*}}^{\mathbb{C}^{*}-1} \times\right) .
\end{aligned}
$$

Lemma 6.5. Fix an element $\xi \in\left(\operatorname{Hilb}^{2}\left(\mathbb{C}^{r-1}, O\right)\right)^{\mathbb{C}^{*}}$. Then,

$$
\sum_{n=0}^{+\infty} \chi\left(\mathfrak{M}_{(2), n ; C_{0}}^{\mathbb{C}^{r-1} \times C}\right) q^{n}=(r-1) \cdot \sum_{n=0}^{+\infty} \chi\left(\mathfrak{M}_{\xi, n ; C_{0}}^{\mathbb{C}^{r-1} \times C}\right) q^{n}
$$

Proof. By (6.1.15) we have

$$
\begin{aligned}
\sum_{n=0}^{+\infty} \chi\left(\mathfrak{M}_{(2), n ; C_{0}}^{\mathbb{C}^{r-1} \times C}\right) q^{n} & =\sum_{n=0}^{+\infty} \sum_{\eta \in\left(\operatorname{Hilb}^{2}\left(\mathbb{C}^{r-1}, O\right)\right)^{\mathbb{C}^{*}}} \chi\left(\mathfrak{M}_{\eta, n ; C_{0}}^{\mathbb{C}^{r-1} \times C}\right) q^{n} \\
& =\sum_{\eta \in\left(\operatorname{Hilb}^{2}\left(\mathbb{C}^{r-1}, O\right)\right)^{\mathbb{C}^{*}}} \sum_{n=0}^{+\infty} \chi\left(\mathfrak{M}_{\eta, n ; C_{0}}^{\mathbb{C}^{r-1} \times C}\right) q^{n} .
\end{aligned}
$$

By (6.1.13), up to isomorphisms, $\mathfrak{M}_{\eta, n ; C_{0}}^{\mathbb{C}^{r-1} \times C}$ is independent of $\eta \in\left(\operatorname{Hilb}^{2}\left(\mathbb{C}^{r-1}, O\right)\right)^{\mathbb{C}^{*}}$. So 


$$
\begin{aligned}
\sum_{n=0}^{+\infty} \chi\left(\mathfrak{M}_{(2), n ; C_{0}}^{\mathbb{C}^{r-1} \times C}\right) q^{n} & =\left|\left(\operatorname{Hilb}^{2}\left(\mathbb{C}^{r-1}, O\right)\right)^{\mathbb{C}^{*}}\right| \cdot \sum_{n=0}^{+\infty} \chi\left(\mathfrak{M}_{\xi, n ; C_{0}}^{\mathbb{C}^{r-1} \times C}\right) q^{n} \\
& =(r-1) \cdot \sum_{n=0}^{+\infty} \chi\left(\mathfrak{M}_{\xi, n ; C_{0}}^{\mathbb{C}^{r-1} \times C}\right) q^{n} .
\end{aligned}
$$

\subsection{Reduction to the punctual cases}

From Proposition 6.3 and Lemma 6.5 we see that it suffices to compute the virtual Hodge polynomials of $\mathfrak{M}_{\xi, n ; C_{0}}^{\mathbb{C}^{r-1} \times C}$ and $\left(\mathbb{C}^{r-1} \times C\right)_{C_{0}}^{[n]}$. These spaces are similar to the Hilbert scheme $X^{[n]}$ in the sense that they are all built up from the punctual cases. Cheah developed a method of computing virtual Hodge polynomials to deal with this kind of situation. In order to apply the method to $\mathfrak{M}_{\xi, n ; C_{0}}^{\mathbb{C}^{r-1} \times C}$ and $\left(\mathbb{C}^{r-1} \times C\right)_{C_{0}}^{[n]}$, we will follow the presentation in Section 6 in [LQ] of Cheah's original approach in [Che] for the case of $X^{[n]}$.

Let $\operatorname{Hilb}^{n}\left(\mathbb{C}^{r}, O\right)$ be the punctual Hilbert scheme of $\mathbb{C}^{r}$ at the origin. Then there exist unique rational numbers $H_{\ell, m, n}$ such that

$$
\sum_{n=0}^{+\infty} e\left(\operatorname{Hilb}^{n}\left(\mathbb{C}^{r}, O\right) ; s, t\right) q^{n}=\prod_{\ell=1}^{+\infty} \prod_{m, n=0}^{+\infty}\left(\frac{1}{1-q^{\ell} s^{m} t^{n}}\right)^{H_{\ell, m, n}}
$$

as elements in $\mathbb{Q}[s, t][[q]]$. Define $\mathfrak{h}_{r}(q, s, t) \in \mathbb{Q}[s, t][[q]]$ to be the power series:

$$
\mathfrak{h}_{r}(q, s, t)=\sum_{\ell=1}^{+\infty}\left(\sum_{m, n=0}^{+\infty} H_{\ell, m, n} s^{m} t^{n}\right) q^{\ell} .
$$

Then the main result proved in [Che] states that

$$
\sum_{n=0}^{+\infty} e\left(X^{[n]} ; s, t\right) q^{n}=\exp \left(\sum_{n=1}^{+\infty} \frac{1}{n} e\left(X ; s^{n}, t^{n}\right) \mathfrak{h}_{r}\left(q^{n}, s^{n}, t^{n}\right)\right) .
$$

The key ingredients in Cheah's proof of (6.2.3) can be summarized as follows: 
(A) Each element $\Xi \in X^{[n]}$ can be uniquely decomposed into $\Xi^{(1)}+\ldots+\Xi^{(\ell)}$ where every $\Xi^{(i)} \in X^{\left[n_{i}\right]}$ is supported at a single point in $X, n_{1}+\ldots+n_{\ell}=n$, and the supports of $\Xi^{(1)}, \ldots, \Xi^{(\ell)}$ are mutually distinct.

(B) Every $X_{x}^{[n]}$ is isomorphic to $\operatorname{Hilb}^{n}\left(\mathbb{C}^{r}, O\right)$. Let $X_{(n)}^{[n]}$ be the closed subscheme of $X^{[n]}$ consisting of all $\Xi \in X^{[n]}$ such that $\operatorname{Supp}(\Xi)$ is a single point of $X$. Then the natural morphism $X_{(n)}^{[n]} \rightarrow X$ sending $\Xi \in X_{(n)}^{[n]}$ to $\operatorname{Supp}(\Xi) \in X$ is Zariski-locally trivial with fibers isomorphic to $\operatorname{Hilb}^{n}\left(\mathbb{C}^{r}, O\right)$.

(C) Using certain combinatorial arguments independent of $X$, one reduces the computation to the virtual Hodge polynomials of $X$ and $\operatorname{Hilb}^{n}\left(\mathbb{C}^{r}, O\right)$ which contribute to the terms $e\left(X ; s^{n}, t^{n}\right)$ and $\mathfrak{h}_{r}\left(q^{n}, s^{n}, t^{n}\right)$ in (6.2.3) respectively.

It follows that we can apply Cheah's arguments to the computations of

$$
\sum_{n=0}^{+\infty} e\left(\left(\mathbb{C}^{r-1} \times C\right)_{C_{0}}^{[n]} ; s, t\right) q^{n}, \quad \sum_{n=0}^{+\infty} e\left(\mathfrak{M}_{\xi, n ; C_{0}}^{\mathbb{C}^{r-1} \times C} ; s, t\right) q^{n}
$$

in a straightforward fashion. For $\sum_{n=0}^{+\infty} e\left(\left(\mathbb{C}^{r-1} \times C\right)_{C_{0}}^{[n]} ; s, t\right) q^{n}$, we have

(A1) Each element $\Xi \in\left(\mathbb{C}^{r-1} \times C\right)_{C_{0}}^{[n]}$ can be uniquely decomposed into

$$
\Xi^{(1)}+\ldots+\Xi^{(\ell)}
$$

where each $\Xi^{(i)} \in\left(\mathbb{C}^{r-1} \times C\right)_{C_{0}}^{\left[n_{i}\right]}$ is supported at a single point in $C_{0}$,

$$
n_{1}+\ldots+n_{\ell}=n,
$$

and the supports of $\Xi^{(1)}, \ldots, \Xi^{(\ell)}$ are mutually distinct. 
(B1) Every $\left(\mathbb{C}^{r-1} \times C\right)_{x}^{[n]}, x \in C_{0}$ is isomorphic to $\operatorname{Hilb}^{n}\left(\mathbb{C}^{r}, O\right)$. The natural morphism

$$
\bigcup_{x \in C_{0}}\left(\mathbb{C}^{r-1} \times C\right)_{x}^{[n]} \rightarrow C_{0}
$$

sending $\Xi \in \bigcup_{x \in C_{0}}\left(\mathbb{C}^{r-1} \times C\right)_{x}^{[n]}$ to $\operatorname{Supp}(\Xi) \in C_{0}$ is Zariski-locally trivial with fibers isomorphic to $\operatorname{Hilb}^{n}\left(\mathbb{C}^{r}, O\right)$.

(C1) The same combinatorial arguments from (C) reduces the computation to the virtual Hodge polynomials of $C_{0}$ and $\operatorname{Hilb}^{n}\left(\mathbb{C}^{r}, O\right)$.

Therefore, we conclude as in (6.2.3) the following formula:

$$
\sum_{n=0}^{+\infty} e\left(\left(\mathbb{C}^{r-1} \times C\right)_{C_{0}}^{[n]} ; s, t\right) q^{n}=\exp \left(\sum_{n=1}^{+\infty} \frac{1}{n} e\left(C_{0} ; s^{n}, t^{n}\right) \mathfrak{h}_{r}\left(q^{n}, s^{n}, t^{n}\right)\right) .
$$

The next lemma is from [LQ].

Lemma 6.6. Let $C_{0}=\{O\} \times C \subseteq \mathbb{C}^{r-1} \times C$. Then,

$$
\sum_{n=0}^{+\infty} \chi\left(\left(\mathbb{C}^{r-1} \times C\right)_{C_{0}}^{[n]}\right) q^{n}=\left(\sum_{n=0}^{+\infty} \chi\left(\operatorname{Hilb}^{n}\left(\mathbb{C}^{r}, O\right)\right) q^{n}\right)^{2-2 g}
$$

Proof. By (3.0.3),

$$
e\left(C_{0} ; 1,1\right)=\chi\left(C_{0}\right)=\chi(C)=2-2 g .
$$

Also, by (6.2.1) and (6.2.2),

$$
\exp \left(\sum_{n=1}^{+\infty} \frac{1}{n} \mathfrak{h}_{r}\left(q^{n}, 1,1\right)\right)=\sum_{n=0}^{+\infty} \chi\left(\operatorname{Hilb}^{n}\left(\mathbb{C}^{r}, O\right)\right) q^{n} .
$$

By (3.0.3) and (6.2.4),

$$
\begin{aligned}
\sum_{n=0}^{+\infty} \chi\left(\left(\mathbb{C}^{r-1} \times C\right)_{C_{0}}^{[n]}\right) q^{n} & =\sum_{n=0}^{+\infty} e\left(\left(\mathbb{C}^{r-1} \times C\right)_{C_{0}}^{[n]} ; 1,1\right) q^{n} \\
& =\exp \left(\sum_{n=1}^{+\infty} \frac{1}{n} e\left(C_{0} ; 1,1\right) \mathfrak{h}_{r}\left(q^{n}, 1,1\right)\right)
\end{aligned}
$$




$$
\begin{aligned}
& =\exp \left(\sum_{n=1}^{+\infty} \frac{1}{n}(2-2 g) \mathfrak{h}_{r}\left(q^{n}, 1,1\right)\right) \\
& =\left(\sum_{n=0}^{+\infty} \chi\left(\operatorname{Hilb}^{n}\left(\mathbb{C}^{r}, O\right)\right) q^{n}\right)^{2-2 g} .
\end{aligned}
$$

Lemma 6.7 below is also from [LQ].

Lemma 6.7. Let $C_{0}=\{O\} \times C \subset \mathbb{C}^{r-1} \times C$. Let $L=\left\{z_{1}=. .=z_{r-1}=0\right\} \subset \mathbb{C}^{r}$. Then,

$$
\sum_{n=0}^{+\infty} \chi\left(\mathfrak{M}_{1, n ; C_{0}}^{\mathbb{C}^{r-1} \times C}\right) q^{n}=\left(\sum_{n=0}^{+\infty} \chi\left(\mathfrak{M}_{1, n ; L, O}^{\mathbb{C}^{r}}\right) q^{n}\right)^{2-2 g}
$$

where $\mathfrak{M}_{1, n ; L, O}^{\mathbb{C}^{r}}$ parametrizes all the 1-dimensional closed subschemes $\Theta$ of $\mathbb{C}^{r}$ such that $I_{\Theta} \subset I_{L}, \operatorname{Supp}\left(I_{L} / I_{\Theta}\right)=\{O\}$, and $h^{0}\left(\mathbb{C}^{r}, I_{L} / I_{\Theta}\right)=n$.

Next, we fix $\xi \in\left(\operatorname{Hilb}^{2}\left(\mathbb{C}^{r-1}, O\right)\right)^{\mathbb{C}^{*}}$. Let $\mathfrak{M}_{\xi, n ; C 0}^{\mathbb{C}^{r-1} \times C}$ be from Definition 6.4. For the computation of $\sum_{n=0}^{+\infty} e\left(\mathfrak{M}_{\xi, n ; C_{0}}^{\mathbb{C}^{r-1} \times C} ; s, t\right) q^{n}$, we have

(A2) Let $\Theta \in \mathfrak{M}_{\xi, n ; C_{0}}^{\mathbb{C}^{r-1} \times C}$. By the definition of $\mathfrak{M}_{\xi, n ; C_{0}}^{\mathbb{C}^{r-1} \times C}$, the quotient $I_{\mu^{*} \xi} / I_{\Theta}$ is supported at finitely many points in $C_{0}$. Put

$$
I_{\mu^{*} \xi} / I_{\Theta}=Q_{1} \oplus \cdots \oplus Q_{\ell}
$$

where each $Q_{i}$ is supported at a single point in $C_{0}$, and the supports of $Q_{1}, \ldots, Q_{\ell}$ are mutually distinct. Let $f: I_{\mu^{*} \xi} \rightarrow I_{\mu^{*} \xi} / I_{\Theta}$ be the quotient map. For $1 \leq i \leq \ell$, define the subscheme $\Theta^{(i)}$ by putting

$$
I_{\Theta^{(i)}}=f^{-1}\left(Q_{i}\right)
$$


Then $\Theta \in \mathfrak{M}_{\xi, n ; C_{0}}^{\mathbb{C}^{r-1} \times C}$ gives rise to $\Theta^{(1)}, \ldots, \Theta^{(\ell)}$. It is clear that the process can be reversed. Hence $\Theta \in \mathfrak{M}_{\xi, n ; C_{0}}^{\mathbb{C}^{r-1} \times C}$ can be formally written as

$$
\Theta=\Theta^{(1)}+\ldots+\Theta^{(\ell)}
$$

in a unique way, where $\Theta^{(i)} \in \mathfrak{M}_{\xi, n_{i} ; C_{0}}^{\mathbb{C}^{r-1} \times C}$ for $1 \leq i \leq \ell, n_{1}+\ldots+n_{\ell}=n$, each quotient $I_{\mu^{*} \xi} / I_{\Theta_{i}}$ is supported at a single point in $C_{0}$, and the supports of the quotients $I_{\mu^{*} \xi} / I_{\Theta_{1}}, \ldots, I_{\mu^{*} \xi} / I_{\Theta_{\ell}}$ are mutually distinct.

(B2) Let $x \in C_{0}$. Since $C$ is a smooth curve in $X$, we have an isomorphism

$$
\mathfrak{M}_{\xi, n ; C_{0}, x}^{\mathbb{C}^{r-1} \times C} \cong \mathfrak{M}_{\xi, n ; L, O}^{\mathbb{C}^{r}}
$$

between the punctual moduli spaces, where

$$
\{L=(0, \ldots, 0, a) \mid a \in \mathbb{C}\} \subset \mathbb{C}^{r}
$$

$O$ is the origin of $\mathbb{C}^{r}$, and $\mathfrak{M}_{\xi, n ; L, O}^{\mathbb{C}^{r}}$ parametrizes all the 1 -dimensional closed subschemes $\Theta$ of $\mathbb{C}^{r}$ such that $I_{\Theta} \subset I_{\mu^{*} \xi}, \operatorname{Supp}\left(I_{\mu^{*} \xi} / I_{\Theta}\right)=\{O\}$, and

$$
h^{0}\left(\mathbb{C}^{r}, I_{\mu^{*} \xi} / I_{\Theta}\right)=n .
$$

Let $\mathfrak{M}_{\xi,(n) ; C_{0}}^{\mathbb{C}^{r-1} \times C}$ be the subset of $\mathfrak{M}_{\xi, n ; C_{0}}^{\mathbb{C}^{r-1} \times C}$ consisting of all $\Theta \in \mathfrak{M}_{\xi, n ; C_{0}}^{\mathbb{C}^{r-1} \times C}$ such that $\operatorname{Supp}\left(I_{\mu^{*} \xi} / I_{\Theta}\right)$ is a single point in $C_{0}$. By the construction in [Gro], there is a natural morphism from $\mathfrak{M}_{2, n}^{\mathbb{C}^{r-1} \times C}$ to the $n$-th symmetric product $\operatorname{Sym}^{n}\left(\mathbb{C}^{r-1} \times\right.$ $C)$. Its restriction to $\mathfrak{M}_{\xi,(n) ; C_{0}}^{\mathbb{C}^{r-1} \times C}$ gives rise to a morphism

$$
\phi: \mathfrak{M}_{\xi,(n) ; C_{0}}^{\mathbb{C}^{r-1} \times C} \rightarrow C_{0}
$$

An argument similar to the proof of Proposition 5.13 shows that there exist a decomposition of locally closed subsets

$$
C_{0}=\coprod_{i} C_{0, i}
$$


and bijective morphisms over the locally closed subsets $C_{0, i}$ :

$$
\Phi_{i}: \mathfrak{M}_{\xi, n ; L, O}^{\mathbb{C}^{r}} \times C_{0, i} \rightarrow \phi^{-1}\left(C_{0, i}\right)
$$

(C2) The same combinatorial arguments from (C) reduces the computation to the virtual Hodge polynomials of $C_{0}$ and $\mathfrak{M}_{\xi, n ; L, O}^{\mathbb{C}^{r}}$

Hence once again, we conclude as in (6.2.3) the following:

$$
\sum_{n=0}^{+\infty} e\left(\mathfrak{M}_{\xi, n ; C_{0}}^{\mathbb{C}^{r-1} \times C} ; s, t\right) q^{n}=\exp \left(\sum_{n=1}^{+\infty} \frac{1}{n} e\left(C_{0} ; s^{n}, t^{n}\right) \mathfrak{c}_{r}\left(q^{n}, s^{n}, t^{n}\right)\right)
$$

where the power series $\mathfrak{c}_{r}(q, s, t) \in \mathbb{Q}[s, t][[q]]$ is defined by

$$
\mathfrak{c}_{r}(q, s, t)=\sum_{\ell=1}^{+\infty}\left(\sum_{m, n=0}^{+\infty} C_{\ell, m, n} s^{m} t^{n}\right) q^{\ell},
$$

and the rational numbers $C_{\ell, m, n}$ are the unique rational numbers such that

$$
\sum_{n=0}^{+\infty} e\left(\mathfrak{M}_{\xi, n ; L, O}^{\mathbb{C}^{r}} ; s, t\right) q^{n}=\prod_{\ell=1}^{+\infty} \prod_{m, n=0}^{+\infty}\left(\frac{1}{1-q^{\ell} s^{m} t^{n}}\right)^{C_{\ell, m, n}} .
$$

Lemma 6.8. Let $C_{0}=\{O\} \times C \subseteq \mathbb{C}^{r-1} \times C$. Then,

$$
\sum_{n=0}^{+\infty} \chi\left(\mathfrak{M}_{\xi, n ; C_{0}}^{\mathbb{C}^{r-1} \times C}\right) q^{n}=\left(\sum_{n=0}^{+\infty} \chi\left(\mathfrak{M}_{\xi, n ; L, O}^{\mathbb{C}^{r}}\right) q^{n}\right)^{2-2 g}
$$

Proof. By (3.0.3),

$$
e\left(C_{0} ; 1,1\right)=\chi\left(C_{0}\right)=\chi(C)=2-2 g .
$$

Also, by (6.2.8) and (6.2.9),

$$
\exp \left(\sum_{n=1}^{+\infty} \frac{1}{n} \mathfrak{c}_{r}\left(q^{n}, 1,1\right)\right)=\sum_{n=0}^{+\infty} \chi\left(\mathfrak{M}_{\xi, n ; L, O}^{\mathbb{C}^{r}}\right) q^{n}
$$

By (3.0.3) and (6.2.7),

$$
\sum_{n=0}^{+\infty} \chi\left(\mathfrak{M}_{\xi, n ; C_{0}}^{\mathbb{C}^{r-1} \times C}\right) q^{n}=\sum_{n=0}^{+\infty} e\left(\mathfrak{M}_{\xi, n ; C_{0}}^{\mathbb{C}^{r-1} \times C} ; 1,1\right) q^{n}
$$




$$
\begin{aligned}
& =\exp \left(\sum_{n=1}^{+\infty} \frac{1}{n} e\left(C_{0} ; 1,1\right) \mathfrak{c}_{r}\left(q^{n}, 1,1\right)\right) \\
& =\exp \left(\sum_{n=1}^{+\infty} \frac{1}{n}(2-2 g) \mathfrak{c}_{r}\left(q^{n}, 1,1\right)\right) \\
& =\left(\sum_{n=0}^{+\infty} \chi\left(\mathfrak{M}_{\xi, n ; L, O}^{\mathbb{C}^{r}}\right) q^{n}\right)^{2-2 g} .
\end{aligned}
$$

Proposition 6.9. Fix $\xi \in\left(\operatorname{Hilb}\left(\mathbb{C}^{r-1}, O\right)\right)^{\mathbb{C}^{*}}$. Let $\mathfrak{M}_{\xi, n ; L, O}^{\mathbb{C}^{r}}$ be from (6.2.6). Under Assumption 5.2, we have

$$
\begin{aligned}
& \sum_{n=0}^{+\infty} \chi\left(\mathfrak{M}_{(2), n}\right) q^{n} \\
= & (r-1) \cdot \sum_{n=0}^{+\infty} \chi\left(X^{[n]}\right) q^{n} \cdot \chi(S) \cdot\left(\frac{\sum_{n=0}^{+\infty} \chi\left(\mathfrak{M}_{\xi, n ; L, O}^{\mathbb{C}^{r}}\right) q^{n}}{\sum_{n=0}^{+\infty} \chi\left(\operatorname{Hilb}^{n}\left(\mathbb{C}^{r}, O\right)\right) q^{n}}\right)^{2-2 g} .
\end{aligned}
$$

Proof. By Proposition 6.3, Lemma 6.5, Lemma 6.6, and Lemma 6.8, we have

$$
\begin{aligned}
& \sum_{n=0}^{+\infty} \chi\left(\mathfrak{M}_{(2), n}\right) q^{n} \\
& =\sum_{n=0}^{+\infty} e\left(\mathfrak{M}_{(2), n} ; 1,1\right) q^{n} \\
& =\sum_{n=0}^{+\infty} e\left(X^{[n]} ; 1,1\right) q^{n} \cdot e(S ; 1,1) \cdot \frac{\sum_{n=0}^{+\infty} e\left(\mathfrak{M}_{(2), n ; C_{0}}^{\mathbb{C}^{r-1}} ; 1,1\right) q^{n}}{\sum_{n=0}^{+\infty} e\left(\left(\mathbb{C}^{r-1} \times C\right)_{C_{0}}^{[n]} ; 1,1\right) q^{n}} \\
& =\sum_{n=0}^{+\infty} e\left(X^{[n]} ; 1,1\right) q^{n} \cdot e(S ; 1,1) \cdot \frac{(r-1) \cdot \sum_{n=0}^{+\infty} \chi\left(\mathfrak{M}_{\xi, n ; C_{0}}^{\mathbb{C}^{r-1} \times C}\right) q^{n}}{\sum_{n=0}^{+\infty} e\left(\left(\mathbb{C}^{r-1} \times C\right)_{C_{0}}^{[n]} ; 1,1\right) q^{n}} \\
& =(r-1) \cdot \sum_{n=0}^{+\infty} \chi\left(X^{[n]}\right) q^{n} \cdot \chi(S) \cdot\left(\frac{\sum_{n=0}^{+\infty} \chi\left(\mathfrak{M}_{\xi, n ; L, O}^{\mathbb{C}^{r}}\right) q^{n}}{\sum_{n=0}^{+\infty} \chi\left(\operatorname{Hilb}^{n}\left(\mathbb{C}^{r}, O\right)\right) q^{n}}\right)^{2-2 g} .
\end{aligned}
$$

\subsection{Torus actions on $\mathfrak{M}_{\xi, n, L, O}^{\mathbb{C}^{r}}$}

According to (3.0.7), we can make use of a suitable $\mathbb{C}^{*}$-action on $\mathbb{C}^{r}$ and count the number of the fixed points of the induced $\mathbb{C}^{*}$-action on $\mathfrak{M}_{\xi, n, L, O}^{\mathbb{C}^{r}}$. The fixed points of 
torus actions on $\mathfrak{M}_{\xi, n, L, O}^{\mathbb{C}^{r}}$ are closely related to multi-dimensional partitions of $n$.

Let $r \geq 3$ and let $z_{1}, z_{2}, \ldots, z_{r}$ be the coordinate functions of $\mathbb{C}^{r}$. Let

$$
L=\left\{z_{1}=\ldots=z_{r-1}=0\right\} \subset \mathbb{C}^{r},
$$

and

$$
\mathbb{C}^{r-1}=\left\{z_{r}=0\right\} \subset \mathbb{C}^{r}
$$

Consider the $\mathbb{C}^{*}$-action $(4.2 .2)$ on $\mathbb{C}^{r}$, and choose the weights $w_{1}, \ldots, w_{r} \in \mathbb{Z}$ in $(4.2 .2)$ properly. Then, we have an induced $\mathbb{C}^{*}$-action on $\mathbb{C}^{r-1} \subset \mathbb{C}^{r}$, and the elements in $\left(\operatorname{Hilb}^{2}\left(\mathbb{C}^{r-1}, O\right)\right)^{\mathbb{C}^{*}}$ are in one-to-one correspondence with the ideals in (6.1.13). Let $\xi \in\left(\operatorname{Hilb}^{2}\left(\mathbb{C}^{r-1}, O\right)\right)^{\mathbb{C}^{*}}$ correspond to the ideal:

$$
I_{\xi}=\left(z_{1}, \ldots, z_{r-2}, z_{r-1}^{2}\right)
$$

Then, we obtain an induced $\mathbb{C}^{*}$-action on $\mathfrak{M}_{\xi, n, L, O}^{\mathbb{C}^{r}}$. The $\mathbb{C}^{*}$-fixed points in $\mathfrak{M}_{\xi, n, L, O}^{\mathbb{C}^{r}}$ are precisely those corresponding to the ideals $I$ generated by monomials such that

$$
I \subset I_{\xi}=\left(z_{1}, \ldots, z_{r-2}, z_{r-1}^{2}\right)
$$

and

$$
\operatorname{dim}_{\mathbb{C}} \frac{\left(z_{1}, \ldots, z_{r-2}, z_{r-1}^{2}\right)}{I}=n
$$

These ideals are in one-to-one correspondence with the $\mathfrak{I}$-punctual $r$-dimensional partitions of $n$ (note that a linear basis of the ideal $\left(z_{1}, \ldots, z_{r-1}, z_{r-1}^{2}\right)$ consists of all the monomials $z_{1}^{i_{1}} \cdots z_{r-1}^{i_{r-1}} z_{r}^{i_{r}}$ with

$$
\left.\left(i_{1}, \ldots, i_{r-1}\right) \in \mathfrak{I}:=\left(\mathbb{Z}_{\geq 0}\right)^{r-1}-\{(0, \ldots, 0),(1,0, \ldots, 0)\}\right)
$$


Indeed, in view of (6.3.1) and (6.3.2), given an $\mathfrak{I}$-punctual $r$-dimensional partition $\left(n_{i_{1}, \ldots, i_{r-1}}\right)_{\left(i_{1}, \ldots, i_{r-1}\right) \in \mathfrak{I}}$ of $n$, the ideal of $\mathbb{C}\left[z_{1}, \ldots, z_{r}\right]$ generated by the monomials $z_{1}^{i_{1}} \cdots z_{r-1}^{i_{r-1}} z_{r}^{n_{i_{1}, \ldots, i_{r-1}}}$ has colength- $n$. Conversely, given a colength- $n$ ideal $I$ of $\mathbb{C}\left[z_{1}, \ldots, z_{r}\right]$ generated by monomials, we obtain an $\mathfrak{I}$-punctual $r$-dimensional partition

$$
\left(n_{i_{1}, \ldots, i_{r-1}}\right)_{\left(i_{1}, \ldots, i_{r-1}\right) \in \mathfrak{I}}
$$

of $n$ by putting

$$
n_{i_{1}, \ldots, i_{r-1}}=\min \left\{i_{r} \mid z_{1}^{i_{1}} \cdots z_{r-1}^{i_{r-1}} z_{r}^{i_{r}} \in I\right\}
$$

Therefore, we obtain from (3.0.7) and Definition 4.1 (iii) that

$$
\chi\left(\mathfrak{M}_{\xi, n, L, O}^{\mathbb{C}^{r}}\right)=A_{r}(n) .
$$

Theorem 6.10. Under Assumption 5.2, let $r \geq 2$. Then,

$$
\sum_{n=0}^{+\infty} \chi\left(\mathfrak{M}_{(2), n}\right) q^{n}=(r-1) \cdot \chi(S) \cdot \sum_{n=0}^{+\infty} \chi\left(X^{[n]}\right) q^{n} \cdot\left(\frac{\sum_{n=0}^{+\infty} A_{r}(n) q^{n}}{\sum_{n=0}^{+\infty} P_{r}(n) q^{n}}\right)^{2-2 g} .
$$

Proof. The formula follows from Proposition 6.9, (4.2.4) and (6.3.4). 


\section{Chapter 7}

\section{The computation of $\chi\left(\mathfrak{M}_{\left(1^{2}\right), n}\right)$}

The following is parallel to Lemma 6.1.

Lemma 7.1. Let $m=\operatorname{dim}(X)-1=r-1$. Let $O$ be the origin of $\mathbb{C}^{m}$ and $C_{0}=$ $\{O\} \times C$. Then,

$$
\sum_{n=0}^{+\infty} \chi\left(\mathfrak{M}_{\left(1^{2}\right), n}\right) q^{n}=\sum_{n=0}^{+\infty} \chi\left(W_{\left(1^{2}\right), n}\right) q^{n} \cdot\left(\sum_{n=0}^{+\infty} \chi\left(\mathfrak{M}_{1, n ; C_{0}}^{\mathbb{C}^{m} \times C}\right) q^{n}\right)^{2} .
$$

Proof. By (5.1.25), (5.1.22), (3.0.4) and (3.0.6), we obtain:

$$
e\left(\mathfrak{M}_{\left(1^{2}\right), n} ; s, t\right)=\sum_{n_{0}=0}^{n} e\left(\mathfrak{M}_{\left(1^{2}\right), n ; n_{0}} ; s, t\right)=\sum_{n_{0}=0}^{n} e\left(W_{\left(1^{2}\right), n-n_{0}} \times_{S_{\left(\left(1^{2}\right)\right)}} \mathfrak{M}_{\left(1^{2}\right), n_{0} ; n_{0}} ; s, t\right) .
$$

By (3.0.3),

$$
\chi\left(\mathfrak{M}_{\left(1^{2}\right), n}\right)=\sum_{n_{0}=0}^{n} \chi\left(W_{\left(1^{2}\right), n-n_{0}} \times_{S^{\left(\left(1^{2}\right)\right)}} \mathfrak{M}_{\left(1^{2}\right), n_{0} ; n_{0}}\right) .
$$

For $0 \leqslant i \leqslant n_{0}$, let

$$
f_{i, n_{0}-i}: \mathfrak{M}_{1, i ; i} \times \mathfrak{M}_{1, n_{0}-i ; n_{0}-i} \rightarrow S \times S=S^{2}
$$

be the product of the natural projections $f_{i}: \mathfrak{M}_{1, i ; i} \rightarrow S$ and

$$
f_{n_{0}-i}: \mathfrak{M}_{1, n_{0}-i ; n_{0}-i} \rightarrow S
$$

from (5.1.9). Let 


$$
\begin{aligned}
\Delta_{S} & =\{(s, s) \mid s \in S\} \subset S \times S \\
S_{0}^{2} & =S \times S-\Delta_{S} .
\end{aligned}
$$

Then we have an unramified morphism

$$
f_{i, n_{0}-i}^{-1}\left(S_{0}^{2}\right) \rightarrow \mathfrak{M}_{\left(1^{2}\right), n_{0} ; n_{0}}
$$

The sum of these morphisms is a $2: 1$ unramified morphism:

$$
f: \coprod_{i=0}^{n_{0}} f_{i, n_{0}-i}^{-1}\left(S_{0}^{2}\right) \rightarrow \mathfrak{M}_{\left(1^{2}\right), n_{0} ; n_{0}}
$$

By Proposition 5.10, there exists a decomposition

$$
S=\coprod S_{i}
$$

of locally closed subsets such that for each pair $(i, j)$, there exists a bijection:

$$
\mathfrak{M}_{1, i ; C_{0}}^{\mathbb{C}^{m} \times C} \times S_{j} \rightarrow f_{i}^{-1}\left(S_{j}\right)
$$

To avoid the notations, we will simply write:

$$
\mathfrak{M}_{1, i ; C_{0}}^{\mathbb{C}^{m} \times C} \times S_{j}=f_{i}^{-1}\left(S_{j}\right)
$$

So we have

$$
\mathfrak{M}_{1, i ; i}=\coprod_{j}\left(\mathfrak{M}_{1, i ; C_{0}}^{\mathbb{C}^{m} \times C} \times S_{j}\right)
$$

and

$$
\mathfrak{M}_{1, i ; i} \times \mathfrak{M}_{1, n_{0}-i ; n_{0}-i}=\coprod_{j_{1}}\left(\mathfrak{M}_{1, i ; C_{0}}^{\mathbb{C}^{m} \times C} \times S_{j_{1}}\right) \times \coprod_{j_{2}}\left(\mathfrak{M}_{1, n_{0}-i ; C_{0}}^{\mathbb{C}^{m} \times C} \times S_{j_{2}}\right)
$$




$$
\begin{aligned}
& =\left(\mathfrak{M}_{1, i ; C_{0}}^{\mathbb{C}^{m} \times C} \times \mathfrak{M}_{1, n_{0}-i ; C_{0}}^{\mathbb{C}^{m} \times C}\right) \times \coprod_{j_{1}, j_{2}}\left(S_{j_{1}} \times S_{j_{2}}\right) \\
& =\left(\mathfrak{M}_{1, i ; C_{0}}^{\mathbb{C}^{m} \times C} \times \mathfrak{M}_{1, n_{0}-i ; C_{0}}^{\mathbb{C}^{m} \times C}\right) \times S^{2} .
\end{aligned}
$$

Under the identification (7.0.6), the morphism $f_{i, n_{0}-i}$ in (7.0.2) is just the second projection:

$$
\left(\mathfrak{M}_{1, i ; C_{0}}^{\mathbb{C}^{m} \times C} \times \mathfrak{M}_{1, n_{0}-i ; C_{0}}^{\mathbb{C}^{m} \times C}\right) \times S^{2} \rightarrow S^{2}
$$

Therefore we obtain

$$
f_{i, n_{0}-i}^{-1}\left(S_{0}^{2}\right)=\left(\mathfrak{M}_{1, i ; C_{0}}^{\mathbb{C}^{m} \times C} \times \mathfrak{M}_{1, n_{0}-i ; C_{0}}^{\mathbb{C}^{m} \times C}\right) \times S_{0}^{2}
$$

It follows that

$$
\coprod_{i=0}^{n_{0}} f_{i, n_{0}-i}^{-1}\left(S_{0}^{2}\right)=\coprod_{i=0}^{n_{0}}\left(\mathfrak{M}_{1, i ; C_{0}}^{\mathbb{C}^{m} \times C} \times \mathfrak{M}_{1, n_{0}-i ; C_{0}}^{\mathbb{C}^{m} \times C}\right) \times S_{0}^{2}
$$

Let

$$
U=\coprod_{i=0}^{n_{0}}\left(\mathfrak{M}_{1, i ; C_{0}}^{\mathbb{C}^{m} \times C} \times \mathfrak{M}_{1, n_{0}-i ; C_{0}}^{\mathbb{C}^{m} \times C}\right) \times S_{0}^{2} .
$$

Now consider the fiber product diagram

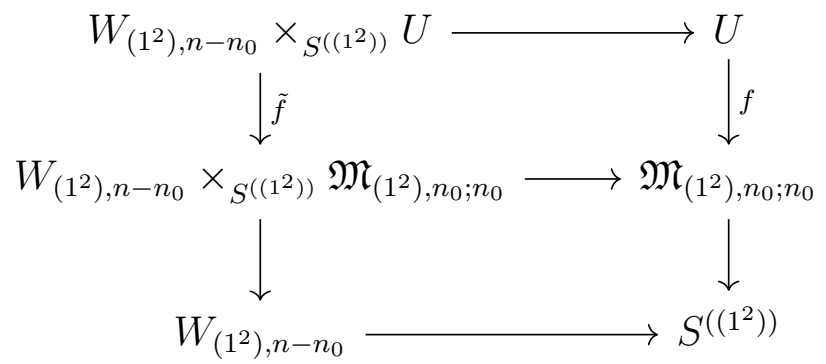

where $\tilde{f}$ is the morphism induced by $f$. Since $f$ is an unramified $2: 1$ map, so is $\tilde{f}$. Therefore by (7.0.1), we have

$$
\begin{aligned}
\chi\left(\mathfrak{M}_{\left(1^{2}\right), n}\right) & =\sum_{n_{0}=0}^{n} \frac{1}{2} \chi\left(W_{\left(1^{2}\right), n-n_{0}} \times_{S_{\left(\left(1^{2}\right)\right)}}\left(\coprod_{i=0}^{n_{0}}\left(\mathfrak{M}_{1, i ; C_{0}}^{\mathbb{C}^{m} \times C} \times \mathfrak{M}_{1, n_{0}-i ; C_{0}}^{\mathbb{C}^{m} \times C}\right) \times S_{0}^{2}\right)\right) \\
& =\sum_{n_{0}=0}^{n} \frac{1}{2} \chi\left(\left(W_{\left(1^{2}\right), n-n_{0}} \times_{S\left(\left(1^{2}\right)\right)} S_{0}^{2}\right) \times \coprod_{i=0}^{n_{0}}\left(\mathfrak{M}_{1, i ; C_{0}}^{\mathbb{C}^{m} \times C} \times \mathfrak{M}_{1, n_{0}-i ; C_{0}}^{\mathbb{C}^{m} \times C}\right)\right)
\end{aligned}
$$




$$
\begin{aligned}
& =\sum_{n_{0}=0}^{n} \frac{1}{2} \chi\left(W_{\left(1^{2}\right), n-n_{0}} \times_{S^{\left(\left(1^{2}\right)\right)}} S_{0}^{2}\right) \cdot \chi\left(\coprod_{i=0}^{n_{0}}\left(\mathfrak{M}_{1, i ; C_{0}}^{\mathbb{C}^{m} \times C} \times \mathfrak{M}_{1, n_{0}-i ; C_{0}}^{\mathbb{C}^{m} \times C}\right)\right) \\
& =\sum_{n_{0}=0}^{n} \frac{1}{2} \chi\left(W_{\left(1^{2}\right), n-n_{0}} \times_{S^{\left(\left(1^{2}\right)\right)}} S_{0}^{2}\right) \cdot \sum_{i=0}^{n_{0}} \chi\left(\mathfrak{M}_{1, i ; C_{0}}^{\mathbb{C}^{m} \times C}\right) \cdot \chi\left(\mathfrak{M}_{1, n_{0}-i ; C_{0}}^{\mathbb{C}^{m} \times C}\right)
\end{aligned}
$$

Since $S_{0}^{2} \rightarrow S^{\left(\left(1^{2}\right)\right)}$ is an unramified $2: 1$ cover,

$$
\chi\left(W_{\left(1^{2}\right), n-n_{0}} \times_{S_{\left(\left(1^{2}\right)\right)}} S_{0}^{2}\right)=2 \chi\left(W_{\left(1^{2}\right), n-n_{0}}\right) .
$$

Therefore

$$
\chi\left(\mathfrak{M}_{\left(1^{2}\right), n}\right)=\sum_{n_{0}=0}^{n} \chi\left(W_{\left(1^{2}\right), n-n_{0}}\right) \cdot \sum_{i=0}^{n_{0}} \chi\left(\mathfrak{M}_{1, i ; C_{0}}^{\mathbb{C}^{m} \times C}\right) \cdot \chi\left(\mathfrak{M}_{1, n_{0}-i ; C_{0}}^{\mathbb{C}^{m} \times C}\right) .
$$

It follows that

$$
\begin{aligned}
& \sum_{n=0}^{+\infty} \chi\left(\mathfrak{M}_{\left(1^{2}\right), n}\right) q^{n} \\
= & \sum_{n=0}^{+\infty} \sum_{n_{0}=0}^{n} \chi\left(W_{\left(1^{2}\right), n-n_{0}}\right) q^{n-n_{0}} \cdot \sum_{i=0}^{n_{0}} \chi\left(\mathfrak{M}_{1, i ; C_{0}}^{\mathbb{C}^{m} \times C}\right) q^{i} \cdot \chi\left(\mathfrak{M}_{1, n_{0}-i ; C_{0}}^{\mathbb{C}^{m} \times C}\right) q^{n_{0}-i} \\
= & \sum_{n=0}^{+\infty} \chi\left(W_{\left(1^{2}\right), n}\right) q^{n} \cdot\left(\sum_{n=0}^{+\infty} \chi\left(\mathfrak{M}_{1, n ; C_{0}}^{\mathbb{C}^{m} \times C}\right) q^{n}\right)^{2}
\end{aligned}
$$

The following is parallel to Lemma 6.2, and its proof is similar to those of Lemma

\section{2 and Lemma 7.1.}

Lemma 7.2. Let $m=\operatorname{dim}(X)-1=r-1$. Let $O$ be the origin of $\mathbb{C}^{m}$ and $C_{0}=$ $\{O\} \times C$. Then,

$$
\sum_{n=0}^{+\infty} \chi\left(X^{[n]} \times S^{\left(\left(1^{2}\right)\right)}\right) q^{n}=\sum_{n=0}^{+\infty} \chi\left(W_{\left(1^{2}\right), n}\right) q^{n} \cdot\left(\sum_{n=0}^{+\infty} \chi\left(\left(\mathbb{C}^{m} \times C\right)_{C_{0}}^{[n]}\right) q^{n}\right)^{2}
$$

Proof. By Definition 5.9,

$$
X^{[n]} \times S^{(\lambda)}=\coprod_{n_{0}=0}^{n} Z_{\lambda, n ; n_{0}}
$$


So

$$
e\left(X^{[n]} \times S^{\left(\left(1^{2}\right)\right)} ; s, t\right)=\sum_{n_{0}=0}^{n} e\left(Z_{\left(1^{2}\right), n ; n_{0}} ; s, t\right) .
$$

By (5.1.23),

$$
e\left(X^{[n]} \times S^{\left(\left(1^{2}\right)\right)} ; s, t\right)=\sum_{n_{0}=0}^{n} e\left(W_{\left(1^{2}\right), n-n_{0}} \times{ }_{S} T_{\left(1^{2}\right), n_{0}} ; s, t\right) .
$$

By an argument similar to that for (7.0.10), we have

$$
\begin{aligned}
& \chi\left(X^{[n]} \times S^{\left(\left(1^{2}\right)\right)}\right) \\
= & \sum_{n_{0}=0}^{n} \chi\left(W_{\left(1^{2}\right), n-n_{0}}\right) \cdot \sum_{i=0}^{n_{0}} \chi\left(\left(\mathbb{C}^{m} \times C\right)_{C_{0}}^{[i]}\right) \cdot \chi\left(\left(\mathbb{C}^{m} \times C\right)_{C_{0}}^{\left[n_{0}-i\right]}\right) .
\end{aligned}
$$

It follows that

$$
\begin{aligned}
& \sum_{n=0}^{+\infty} \chi\left(X^{[n]} \times S^{\left(\left(^{2}\right)\right)}\right) q^{n} \\
= & \sum_{n=0}^{+\infty} \sum_{n_{0}=0}^{n} \chi\left(W_{\left(1^{2}\right), n-n_{0}}\right) q^{n-n_{0}} \cdot \sum_{i=0}^{n_{0}} \chi\left(\left(\mathbb{C}^{m} \times C\right)_{C_{0}}^{[i]}\right) q^{i} \cdot \chi\left(\left(\mathbb{C}^{m} \times C\right)_{C_{0}}^{\left[n_{0}-i\right]}\right) q^{n_{0}-i} \\
= & \sum_{n=0}^{+\infty} \chi\left(W_{\left(1^{2}\right), n}\right) q^{n} \cdot\left(\sum_{n=0}^{+\infty} \chi\left(\left(\mathbb{C}^{m} \times C\right)_{C_{0}}^{[n]}\right) q^{n}\right)^{2}
\end{aligned}
$$

The following is parallel to Theorem 6.10.

Theorem 7.3. Under Assumption 5.2, let $r \geq 2$. Then,

$$
\sum_{n=0}^{+\infty} \chi\left(\mathfrak{M}_{\left(1^{2}\right), n}\right) q^{n}=\frac{\chi(S)^{2}-\chi(S)}{2} \cdot \sum_{n=0}^{+\infty} \chi\left(X^{[n]}\right) q^{n} \cdot\left(\frac{\sum_{n=0}^{+\infty} \widetilde{P}_{r}(n) q^{n}}{\sum_{n=0}^{+\infty} P_{r}(n) q^{n}}\right)^{4-4 g}
$$

Proof. Let $m=\operatorname{dim}(X)-1=r-1$. By Lemma 7.1 and Lemma 7.2,

$$
\sum_{n=0}^{+\infty} \chi\left(\mathfrak{M}_{\left(1^{2}\right), n}\right) q^{n}=\sum_{n=0}^{+\infty} \chi\left(X^{[n]} \times S^{\left(\left(1^{2}\right)\right)}\right) q^{n} \cdot\left(\frac{\sum_{n=0}^{+\infty} \chi\left(\mathfrak{M}_{1, n ; C_{0}}^{\mathbb{C}^{m}} \times q^{n}\right.}{\sum_{n=0}^{+\infty} \chi\left(\left(\mathbb{C}^{m} \times C\right)_{C_{0}}^{[n]}\right) q^{n}}\right)^{2}
$$


where $C_{0}=\{O\} \times C \subset \mathbb{C}^{m} \times C$. Recall $S_{0}^{2}$ from (7.0.4). Since $S_{0}^{2} \rightarrow S^{\left(\left(1^{2}\right)\right)}$ is an unramified $2: 1$ cover,

$$
\begin{aligned}
\chi\left(X^{[n]} \times S^{\left(\left(1^{2}\right)\right)}\right) & =\chi\left(X^{[n]}\right) \cdot \chi\left(S^{\left(\left(1^{2}\right)\right)}\right) \\
& =\frac{1}{2} \cdot \chi\left(X^{[n]}\right) \cdot \chi\left(S_{0}^{2}\right) \\
& =\frac{1}{2} \cdot \chi\left(X^{[n]}\right) \cdot \chi\left(S^{2}-\Delta_{S}\right) \\
& =\frac{1}{2} \cdot \chi\left(X^{[n]}\right) \cdot\left(\chi(S)^{2}-\chi(S)\right) .
\end{aligned}
$$

Thus, we have

$$
\begin{aligned}
& \sum_{n=0}^{+\infty} \chi\left(\mathfrak{M}_{\left(1^{2}\right), n}\right) q^{n} \\
= & \frac{\chi(S)^{2}-\chi(S)}{2} \cdot \sum_{n=0}^{+\infty} \chi\left(X^{[n]}\right) q^{n} \cdot\left(\frac{\sum_{n=0}^{+\infty} \chi\left(\mathfrak{M}_{1, n ; C_{0}}^{\mathbb{C}^{m} \times C}\right) q^{n}}{\sum_{n=0}^{+\infty} \chi\left(\left(\mathbb{C}^{m} \times C\right)_{C_{0}}^{[n]}\right) q^{n}}\right)^{2} .
\end{aligned}
$$

Combining with Lemma 6.6 and Lemma 6.7, we obtain

$$
\begin{aligned}
& \sum_{n=0}^{+\infty} \chi\left(\mathfrak{M}_{\left(1^{2}\right), n}\right) q^{n} \\
= & \frac{\chi(S)^{2}-\chi(S)}{2} \cdot \sum_{n=0}^{+\infty} \chi\left(X^{[n]}\right) q^{n} \cdot\left(\frac{\sum_{n=0}^{+\infty} \chi\left(\mathfrak{M}_{1, n ; L, O}^{\mathbb{C}^{r}}\right) q^{n}}{\sum_{n=0}^{+\infty} \chi\left(\operatorname{Hilb}^{n}\left(\mathbb{C}^{r}, O\right)\right) q^{n}}\right)^{4-4 g}
\end{aligned}
$$

where $L=\left\{z_{1}=. .=z_{r-1}=0\right\} \subset \mathbb{C}^{r}$ and $O$ is the origin of $\mathbb{C}^{r}$. By formula (7.7) in $[\mathrm{LQ}], \chi\left(\mathfrak{M}_{1, n ; L, O}^{\mathbb{C}^{r}}\right)=\widetilde{P}_{r}(n)$. By $(4.2 .4), \chi\left(\operatorname{Hilb}^{n}\left(\mathbb{C}^{r}, O\right)\right)=P_{r}(n)$. It follows that

$$
\sum_{n=0}^{+\infty} \chi\left(\mathfrak{M}_{\left(1^{2}\right), n}\right) q^{n}=\frac{\chi(S)^{2}-\chi(S)}{2} \cdot \sum_{n=0}^{+\infty} \chi\left(X^{[n]}\right) q^{n} \cdot\left(\frac{\sum_{n=0}^{+\infty} \widetilde{P}_{r}(n) q^{n}}{\sum_{n=0}^{+\infty} P_{r}(n) q^{n}}\right)^{4-4 g}
$$




\section{Chapter 8}

\section{Euler characteristics of $\mathfrak{M}_{2, n}$}

In this chapter we sum up the previous results to compute the Euler characteristics of $\mathfrak{M}_{2, n}$.

Theorem 8.1. Under Assumption 5.2, let $r \geqslant 2$. Then,

$$
\begin{aligned}
& \sum_{n=0}^{+\infty} \chi\left(\mathfrak{M}_{2, n}\right) q^{n} \\
= & \frac{\chi(S)^{2}-\chi(S)}{2} \cdot \sum_{n=0}^{+\infty} \chi\left(X^{[n]}\right) q^{n} \cdot\left(\frac{\sum_{n=0}^{+\infty} \widetilde{P}_{r}(n) q^{n}}{\sum_{n=0}^{+\infty} P_{r}(n) q^{n}}\right)^{4-4 g} \\
& +(r-1) \cdot \chi(S) \cdot \sum_{n=0}^{+\infty} \chi\left(X^{[n]}\right) q^{n} \cdot\left(\frac{\sum_{n=0}^{+\infty} A_{r}(n) q^{n}}{\sum_{n=0}^{+\infty} P_{r}(n) q^{n}}\right)^{2-2 g} .
\end{aligned}
$$

Proof. By (3.0.3), (3.0.4), (5.1.24), Theorem 7.3 and Theorem 6.10, we have

$$
\begin{aligned}
& \sum_{n=0}^{+\infty} \chi\left(\mathfrak{M}_{2, n}\right) q^{n} \\
= & \sum_{n=0}^{+\infty} \chi\left(\mathfrak{M}_{\left(1^{2}\right), n}\right) q^{n}+\sum_{n=0}^{+\infty} \chi\left(\mathfrak{M}_{(2), n}\right) q^{n} \\
= & \frac{\chi(S)^{2}-\chi(S)}{2} \cdot \sum_{n=0}^{+\infty} \chi\left(X^{[n]}\right) q^{n} \cdot\left(\frac{\sum_{n=0}^{+\infty} \widetilde{P}_{r}(n) q^{n}}{\sum_{n=0}^{+\infty} P_{r}(n) q^{n}}\right)^{4-4 g} \\
& +(r-1) \cdot \chi(S) \cdot \sum_{n=0}^{+\infty} \chi\left(X^{[n]}\right) q^{n} \cdot\left(\frac{\sum_{n=0}^{+\infty} A_{r}(n) q^{n}}{\sum_{n=0}^{+\infty} P_{r}(n) q^{n}}\right)^{2-2 g} .
\end{aligned}
$$


Remark 8.2. The generating function of the Euler characteristics of $\chi\left(X^{[n]}\right)$ of the Hilbert scheme $X^{[n]}$ was calculated in [Che, ES] (see also [Qin]):

$$
\sum_{n=0}^{+\infty} \chi\left(X^{[n]}\right) q^{n}=\left(\sum_{n=0}^{+\infty} P_{r}(n) q^{n}\right)^{\chi(X)}
$$

Corollary 8.3. Under Assumption 5.2, let $r \geqslant 2$ and $g=1$. Then,

$$
\sum_{n=0}^{+\infty} \chi\left(\mathfrak{M}_{2, n}\right) q^{n}=\frac{\chi(S)^{2}+(2 r-3) \cdot \chi(S)}{2} \cdot\left(\sum_{n=0}^{+\infty} P_{r}(n) q^{n}\right)^{\chi(X)} .
$$

Proof. By Theorem 8.1,

$$
\sum_{n=0}^{+\infty} \chi\left(\mathfrak{M}_{2, n}\right) q^{n}=\frac{\chi(S)^{2}+(2 r-3) \cdot \chi(S)}{2} \cdot \sum_{n=0}^{+\infty} \chi\left(X^{[n]}\right) q^{n}
$$

By Remark 8.2,

$$
\sum_{n=0}^{+\infty} \chi\left(\mathfrak{M}_{2, n}\right) q^{n}=\frac{\chi(S)^{2}+(2 r-3) \cdot \chi(S)}{2} \cdot\left(\sum_{n=0}^{+\infty} P_{r}(n) q^{n}\right)^{\chi(X)}
$$

Corollary 8.4. Under Assumption 5.2, for $r=3$ and $|q|<1 / 3$, we have

$$
\begin{aligned}
& \sum_{n=0}^{+\infty} \chi\left(\mathfrak{M}_{2, n}\right) q^{n} \\
= & \frac{\chi(S)^{2}-\chi(S)}{2} \cdot\left(\prod_{n=1}^{+\infty} \frac{1}{\left(1-q^{n}\right)^{n}}\right)^{\chi(X)} \cdot \frac{1}{(1-q)^{4-4 g}} \\
& +2 \chi(S) \cdot\left(\prod_{n=1}^{+\infty} \frac{1}{\left(1-q^{n}\right)^{n}}\right)^{\chi(X)} \cdot \frac{1}{\left((1-q)\left(1-q^{2}\right)\right)^{2-2 g}} .
\end{aligned}
$$

Proof. By Theorem 8.1 and Remark 8.2 we have

$$
\begin{aligned}
& \sum_{n=0}^{+\infty} \chi\left(\mathfrak{M}_{2, n}\right) q^{n} \\
= & \frac{\chi(S)^{2}-\chi(S)}{2} \cdot\left(\sum_{n=0}^{+\infty} P_{r}(n) q^{n}\right)^{\chi(X)} \cdot\left(\frac{\sum_{n=0}^{+\infty} \widetilde{P}_{r}(n) q^{n}}{\sum_{n=0}^{+\infty} P_{r}(n) q^{n}}\right)^{4-4 g}
\end{aligned}
$$




$$
+(r-1) \cdot \chi(S) \cdot\left(\sum_{n=0}^{+\infty} P_{r}(n) q^{n}\right)^{\chi(X)} \cdot\left(\frac{\sum_{n=0}^{+\infty} A_{r}(n) q^{n}}{\sum_{n=0}^{+\infty} P_{r}(n) q^{n}}\right)^{2-2 g} .
$$

By (4.2.6) and (4.2.7) we get

$$
\begin{aligned}
& \sum_{n=0}^{+\infty} \chi\left(\mathfrak{M}_{2, n}\right) q^{n} \\
= & \frac{\chi(S)^{2}-\chi(S)}{2} \cdot\left(\prod_{n=1}^{+\infty} \frac{1}{\left(1-q^{n}\right)^{n}}\right)^{\chi(X)} \cdot \frac{1}{(1-q)^{4-4 g}} \\
& +(r-1) \cdot \chi(S) \cdot\left(\prod_{n=1}^{+\infty} \frac{1}{\left(1-q^{n}\right)^{n}}\right)^{\chi(X)} \cdot\left(\frac{\sum_{n=0}^{+\infty} A_{r}(n) q^{n}}{\sum_{n=0}^{+\infty} P_{r}(n) q^{n}}\right)^{2-2 g} .
\end{aligned}
$$

Finally, the result follows by Proposition 4.4. 


\section{Bibliography}

[And] G. Andrews, The theory of partitions, Encyclopedia of Mathematics and its Applications (1976).

[Che] J. Cheah, On the cohomology of Hilbert schemes of points, J. Alg. Geom. 5 (1996), 479-511.

[DK] V.I. Danilov, A.G. Khovanskii, Newton polyhedra and an algorithm for computing Hodge-Deligne numbers, Math. USSR Izv. 29 (1987), 279-298.

[Del] P. Deligne, Théorie de Hodge III, I.H.E.S. Publ. Math. 44 (1974), 5-77.

[DT] S.K. Donaldson, R.P. Thomas, Gauge theory in higher dimensions, In: The geometric universe (Oxford, 1996), 31-47. Oxford Univ. Press, Oxford, 1998.

[ES] G. Ellingsrud, S.A. Strømme, On the homology of the Hilbert scheme of points in the plane. Invent. Math. 87 (1987), 343-352.

[Ful] W. Fulton, Introduction to toric varieties, Annals of Mathematics Studies 131. Princeton University Press, Princeton, 1993.

[Go1] L. Göttsche, The Betti numbers of the Hilbert scheme of points on a smooth projective surface, Math. Ann. 286 (1990), 193-207.

[Go2] L. Göttsche, Hilbert schemes of zero-dimensional subschemes of smooth varieties, Lecture Notes in Mathematics 1572. Springer-Verlag, Berlin, 1994.

[Gro] A. Grothendieck, Techniques de construction et théorèmes d'existence en géométrie algébrique IV, Sem. Bourbaki 221, 13. 1960-1961.

[HM] J. Harris, I. Morrison, Moduli of Curves. New York: Springer, 1998. Print.

[HL] D. Huybrechts, M. Lehn, The geometry of moduli spaces of sheaves, Aspects of Mathematics, E31. Friedr. Vieweg \& Sohn, Braunschweig, 1997.

[KLQ] S. Katz, W. Li, Z. Qin, On The Euler Number of Moduli Spaces of Ideal Sheaves and Donaldson-Thomas Invariants, Math. Res. Letters 14 (2007), 403-411. 
[KM] J. Koslowski, A. Melton, Categorical Perspectives. Boston: Birkhauser, (2001). Print.

[LY] H.B. Lawson, Jr., S.S.T. Yau, Holomorphic symmetries, Ann. Sci. Ecole Norm. Sup., $4^{\mathrm{e}}$ série, t. 20 (1987), 557-577.

[LQ] W. Li, Z. Qin, On The Euler Number of Moduli Spaces of Curves and Points, Commu. in Anal. and Geom. 14 (2006), 387-410.

[MNOP1] D. Maulik, N. Nekrasov, A. Okounkov, R. Pandharipande, GromovWitten theory and Donaldson-Thomas theory, , Compos. Math. 142 (2006), 1263-1285.

[MNOP2] D. Maulik, N. Nekrasov, A. Okounkov, R. Pandharipande, GromovWitten theory and Donaldson-Thomas theory, II, Compos. Math. 142 (2006), 1286-1304.

[Mil] J.S. Milne, Étale cohomology. Princeton Math. Series 33. Princeton: Princeton University Press 1980.

[Nit] N. Nitsure, Construction of Hilbert and Quot Schemes. arXiv:math/0504590v1 [math.AG]

[Qin] Z. Qin, Hilbert schemes of points and infinite dimensional Lie algebras.A book to appear in the Mathematical Surveys and Monographs series, American Mathematical Society.

[Tho] R.P. Thomas, A holomorphic Casson invariant for Calabi-Yau 3-folds, and bundles on K3 fibrations, J. Differential Geom. 54 (2000), 367-438. 
Mazen M. Alhwaimel was born in January 15, 1986 in city of Buraidah, Saudi Arabia. He attended prince Sultan bin Abdulaziz educational complex for his high school. Then, he spent the first two years in Qassim university for his undergraduate study, after that he received a full scholarship to finish his studies in mathematics at University of Missouri-Columbia. After graduation, he was appointed for a teacher assistant position in math department at Qassim university. In 2011, he received a full scholarship to pursue his MA and $\mathrm{PhD}$ under supervision of professor Zhenbo Qin at MU. He is expected to defend his thesis in Fall 2017. 